\title{
Computation of the Longitudinal and Transverse Dispersion Coefficient in an Adsorbing Porous Medium Using Homogenization
}

\author{
Hans Bruining • Mohamed Darwish • Aiske Rijnks
}

Received: 31 March 2009 / Accepted: 8 September 2011 / Published online: 7 October 2011

(C) The Author(s) 2011. This article is published with open access at Springerlink.com

\begin{abstract}
This article compares for the first time, local longitudinal and transverse dispersion coefficients obtained by homogenization with experimental data of dispersion coefficients in porous media, using the correct porosity dependence. It is shown that the longitudinal dispersion coefficient can be reasonably represented by a simple periodic unit cell (PUC), which consists of a single sphere in a cube. We present a slightly modified and simplified approach to derive the homogenized equations, which emphasizes physical aspects of homogenization. Subsequently, we give full dimensional expressions for the dispersion tensor based on a comparison with the convective dispersion equation used for contaminant transport, inclusive the correct dependence on porosity. For the PUC of choice, the dispersion relations are identical to the relations obtained for periodic media. We show that commercial finite element software can be readily used to compute longitudinal and transverse dispersion coefficients in 2D and 3D. The 3D results are for the first time obtained at relevant Peclet numbers. There is good agreement for longitudinal dispersion. The computed transverse dispersion coefficients for a single sphere in a cube are much too low. The effect of adsorption on the dispersion coefficient is also studied. Adsorption does not affect the transverse dispersion coefficient. However, adsorption enhances the longitudinal dispersion coefficient in agreement with an analysis of homogenization applied to Taylor dispersion discussed in the literature.
\end{abstract}

H. Bruining $(\bowtie)$

Section of Geo-Engineering, Faculty of Civil Engineering and Geosciences,

TU Delft, Stevinweg 1, 2628 CN Delft, The Netherlands

e-mail: j.bruining@tudelft.nl

M. Darwish

Shell Exploration \& Production International Centre, Kessler Park 1, 2288 GS Rijswijk, The Netherlands e-mail: Mohamed.IM.Darwish@ shell.com

A. Rijnks

Statoil ASA, Sandslihaugen 30, Bergen, Norway

e-mail: AIRIJ@statoil.com 
Keywords Homogenization - Dispersion tensor - Adsorption

\section{List of Symbols}

\begin{tabular}{|c|c|}
\hline$c(x, y, z, t)$ & Tracer concentration \\
\hline$c_{\mathrm{a}}$ & Equilibrium surface concentration \\
\hline$c_{\mathrm{S}}$ & Absorbed concentration (Eq. 2.6) \\
\hline$c^{(n)}$ & See Eq. 2.7 \\
\hline$D$ & Longitudinal or transverse component of $\mathbf{D}$ \\
\hline $\mathbf{D}^{\mathrm{m}}$ & Effective molecular diffusion tensor \\
\hline $\mathbf{D}^{\mathrm{d}}$ & Hydrodynamic dispersion tensor \\
\hline$D_{0}$ & Molecular diffusion coefficient \\
\hline$D_{x x}^{\mathrm{d}}, D_{x x}^{\mathrm{m}}$ & Longitudinal component of $\mathbf{D}^{\mathrm{d}}$ and $\mathbf{D}^{\mathrm{m}}$ \\
\hline$D_{y y}^{\mathrm{d}}, D_{y y}^{\mathrm{m}}$ & Transverse component of $\mathbf{D}^{\mathrm{d}}$ and $\mathbf{D}^{\mathrm{m}}$ \\
\hline $\mathbf{D}^{y-2}$ & $\mathbf{D}^{\mathrm{m}}+\mathbf{D}^{\mathrm{d}}$ \\
\hline$K$ & Distribution coefficient \\
\hline$L$ & Characteristic macroscopic length \\
\hline$\ell$ & Characteristic length of the PUC \\
\hline$N$ & Number of dimensions \\
\hline$n$ & Outward unit normal \\
\hline PUC & Periodic unit cell \\
\hline$p$ & Pressure \\
\hline$P e$ & Peclet number \\
\hline$\langle Q\rangle$ & Average of quantity $Q$ \\
\hline$Q_{\mathrm{R}}, Q_{\mathrm{D}}$ & Reference and dimensionless quantity \\
\hline$R$ & Retardation \\
\hline $\mathbf{r}_{\mathrm{b}}$ & Big scale (global) coordinate \\
\hline $\mathbf{r}_{\mathrm{s}}$ & Small scale (local) coordinate \\
\hline$t$ & Time \\
\hline$u$ & Darcy velocity \\
\hline$u_{\text {inj }}$ & Darcy injection velocity \\
\hline$\overline{\mathbf{u}}$ & Average Darcy velocity vector \\
\hline$v$ & Fluid velocity \\
\hline$\overline{\mathbf{v}}$ & Average interstitial velocity vector \\
\hline$v_{\mathrm{R}}$ & Reference velocity \\
\hline$\Gamma$ & Grain boundary \\
\hline$\delta$ & Thickness of sorption layer \\
\hline$\partial \Omega$ & Outer boundary of PUC \\
\hline$\varepsilon$ & Scaling parameter $\ell / L \ll 1$ \\
\hline$\mu$ & Viscosity \\
\hline$\varphi$ & Porosity \\
\hline$\vec{x}$ & $c^{(1)}=\overrightarrow{\vec{\chi}} \cdot \operatorname{grad}_{\mathrm{b}} c^{(0)}$ \\
\hline$\chi_{x}$ & $x$-Component of $\vec{\chi}$ \\
\hline$\Omega$ & Total domain of PUC \\
\hline$\Omega_{1}$ & Fluid domain in the PUC \\
\hline$\Omega_{\mathrm{m}}$ & Grain domain in the PUC \\
\hline
\end{tabular}




\section{Introduction}

Reactive transport in porous media plays an important role in environmental hydrology, petroleum engineering, and agricultural engineering. Our interest in upscaling methods was motivated by the desire to interpret laboratory experiments (Darwish et al. 2006) related to Arsenic (As) remediation processes for drinking water in Bangladesh and Bihar (India) (Bhatt 2011); if $\mathrm{Fe}^{2+}$ is deposited on the sand grains it shields the As adsorbing $\mathrm{Fe}^{\mathrm{III}}$ oxides that cover the grains; this leads to arsenic production in the drinking water wells. The article by Bouddour et al. (1996) about upscaling deposition and erosion phenomena by homogenization in porous media was in particular appealing because it describes interesting new mechanisms, such as surface dispersion deposition and surface dispersion erosion. This article, however, does not as yet incorporate the computation of these coefficients. Another appealing aspect of homogenization (Hornung 1997; Mikelic and Rosier 2004; Sanchez-Palencia 1980) is that it does not need a closure relation for obtaining the macroscale transport equation as opposed to volume averaging (Wood 2009).

However, the relevant model equations are not conventional and not easy to solve (Tardif d'Hamonville et al. 2007). There are only very few references (Tardif d'Hamonville et al. 2007) dealing with the computation of one of the transport terms, i.e., the dispersion tensor, and in the 3D setting only results are obtained for low Peclet numbers $(P e)$. Carbonnell and Whitaker (1983) combine a derivation by Brenner (1980) for periodic media with volume averaging and show that they obtain an equation that is identical to the equation obtained with homogenization, i.e., Eq. 3.13. Edwards et al. (1991) and many other authors (Didierjean 1997; Eidsath et al. 1983; Souto and Moyne 1997) use this equation for periodic media and present $2 \mathrm{D}$ computational results also for higher Peclet numbers. The comparison with experimental values uses a standard plot of the longitudinal dispersion coefficient divided by the molecular diffusion coefficient versus the Peclet number (Arya et al. 1988). A dissimilar definition of the dispersion tensor by the engineering and mathematics community, differing by a factor equal to the porosity, precluded until now a correct comparison between experimental and theoretical values obtained with homogenization (Tardif d'Hamonville et al. 2007). This factor is, however, correctly incorporated in the periodic media literature (Edwards et al. 1991).

One purpose of this article is to show that the solution of the model equations can be obtained by software that is readily available and give a comparison between experimental and theoretical values. We have limited the scope of this article to the computation of the dispersion tensor including equilibrium adsorption at the grain surface (Auriault and Lewandowska 1993, 1997; Mauri 1991). It is expected that the next step, i.e., the computation of the surface dispersion deposition and surface erosion coefficients is a logical extension of the results obtained in this article. It follows from the problem statement above that of the many articles of Auriault, we use article (Bouddour et al. 1996) as a key-reference for the derivations. There are, however, more than 70 articles and a book (Auriault et al. 2009) coauthored by Auriault that deal with similar aspects (Auriault 2002; Auriault and Adler 1995; Auriault et al. 1992, 2005; Auriault and Lewandowska 1993, 1996, 1997, 2001; Auriault and Royer 1993a,b). Moreover, there are new developments (Allaire et al. 2010a,b) that concern homogenization for reactive flow in a moving frame of reference.

A few remarks are relevant to put the engineering application in the appropriate context. Lowe and Frenkel (1996) simulate dispersion in a random lattice Boltzmann system by using the integral of the Velocity Auto Correlation Function with respect to time. It is found that this integral does not converge and this would suggest that the ensuing longitudinal convective dispersion coefficient increases with time. This puts the fundamental validity of the 
dispersion phenomenon into question. No convergence problems occur, however, for dispersion coefficients computed with homogenization or in periodic media. The behavior of the dispersion coefficient in the transition region from molecular diffusion to a completely dispersion dominated system has been analyzed for periodic media in Bensoussan et al. (1978), Bhattacharya and Gupta (1983), Bhattacharya et al. (1989), and Gupta and Bhattacharya (1986). We distinguish between upscaling from the pore-scale to the core scale (local dispersion) and from the core scale to the field scale (macroscopic dispersion) (Gelhar 1993). We ignore all the scales below the pore-scale (see however, Bhattacharya et al. 1989). For interpretation of laboratory experiments core-scale equations are relevant. Still, for upscaling to the field scale macroscopic dispersion needs to be considered in practical applications to arsenic remediation, but this is beyond the scope of this article. Attinger et al. (1988) use homogenization theory to scale from the core scale to the field scale. They obtain an average adsorption isotherm by using that on the core scale the concentrations are constant. Also, it was assumed that the local Peclet number is of the order unity with respect to the upscaling factor. Other examples of articles that address the influence of adsorption on dispersion are Attinger et al. (1988), Chrysikopoulos et al. (1992), Moralles-Wilhelm and Gelhar (1996), Rajaram and Gelhar (1993, 1995), Roth and Roth (1993), Van Genuchten and Wierenga (1976), and Uffink et al. (2011) and references cited therein. In this article, we will apply homogenization to an adsorption-convection-diffusion process in porous media for upscaling from the pore-scale to the core scale, leading to an upscaled equation that can be used for the interpretation of laboratory experiments.

An often mentioned criticism toward homogenization is the apparent central role played by periodic boundary conditions, which are considered physically unrealistic. The question is whether effective properties converge as the size of the periodic unit cell (PUC) increases while keeping the upscaling factor the same order of magnitude. Bourgeat and Piatnitski (2004) show that, if separation of scale is possible, effective properties in random media converge as the scale of the unit cell increases, independent of its boundary conditions (periodic, Dirichlet, or Neumann). For the same reason the method of volume averaging (Bachmat and Bear 1983; Bourgeat et al. 1988; Marle 1982; Whitaker 1999) also uses periodic boundary conditions to obtain transport coefficients (Wood 2009).

The question that remains is whether a very simple PUC, like a single grain in a symmetry element is indeed able to find representative values of the dispersion coefficients. Auriault and Lewandowska (1996) state that although the assumption of a periodic structure is not realistic, it is found to adequately model real situations. Brenner (1980), who pioneered the use of periodic models to describe behavior in porous media, points out that a porous medium is neither periodic nor random. Durlofsky (1991) states that in the absence of more detailed knowledge and in the absence of large fluctuations on the unit cell scale, periodic boundary conditions are a reasonable means to obtain up-scaled permeabilities. He applies this upscaling to obtain effective properties on the global scale and also uses this as a starting point to obtain global boundary conditions until convergence is obtained. In this way, it is possible to get useful results in the absence of the possibility of a complete separation of scales in the sense described by Bourgeat and Piatnitski (2004). In fractured media the use of a too small PUC cannot be avoided, but it is still possible to get insight in the fracture-matrix transfer process (Salimi and Bruining 2010a,b).

This article addresses a number of aspects that are not fully covered in the articles dealing with homogenization to derive transport coefficients: (1) it explains the physics behind each step in the derivations, (2) it uses a method familiar to engineers and proposed in Shook et al. (1992) to introduce dimensionless quantities, (3) it shows that the numerical model equations also in 3D can be easily solved with standard finite element software packages; 
this greatly enhances the applicability of homogenization to the engineering practice, (4) the article gives the correct porosity dependence in the expressions for longitudinal dispersion using the definition common in the engineering literature (Bear 1972); it shows for the first time that, as to homogenization, the simplest possible unit cell gives good agreement between the computed and measured longitudinal dispersion coefficients, and (5) it shows that equilibrium adsorption on the grain enhances longitudinal dispersion. Also, Van Duijn et al. (2008) show that the introduction of adsorption enhances the dispersion coefficient in Taylor dispersion.

In our nomenclature, we follow Perkins and Dallas (1962) and distinguish the effective diffusion coefficient $D_{x x, m}$, which is the molecular diffusion coefficient corrected for tortuosity effects, i.e., $D_{x x, m}=D_{0} / \tau$, the total longitudinal (transverse) dispersion coefficient, which consists of a sum of a contribution from the effective diffusion coefficient and from the longitudinal (transverse) convective dispersion coefficient (Dagan and Neuman 1997; Gelhar 1993; Rubin and Hubbard 2005; Zhang 2002) ( $\left.D_{x x, m}, D_{y y, d}\right)$.

The Appendices A, B, and C describe details of the derivations. Section 2 describes homogenization and Sect. 3 derives the up-scaled equations. Section 4 gives an explicit derivation of the longitudinal and transverse dispersion coefficients. Section 5 deals with the numerical implementation of the ensuing model equations in commercial finite element software (COMSOL). Section 6 makes a comparison between homogenization data and both experimental data and some theoretical results from periodic media in the literature. We end with some conclusions.

\section{Equations on the Microscale}

The microscopic system, e.g., a collection of grains, is embedded in a domain of macroscopic (global) scale dimensions with a length $L$. This domain represents the core scale. In the global setting, we use Darcy's law and an upscaled version of the convection-diffusion equation with adsorption that is derived in this article. We apply a potential gradient on the large scale. This leads to an average interstitial velocity $v_{\mathrm{R}}$ equal to the Darcy velocity divided by the porosity. An inert tracer moves due to this velocity field and is subjected to diffusion and adsorption. The velocity will be used to define the Peclet number (see below). We also specify the injection concentration $c_{0}$ and the outflow boundary condition $D \partial c / \partial n=0$. In our equations, we will use the relative concentration by dividing by $c_{0}$ and denote it as the concentration $c$. The outflow boundary does not introduce further characteristic quantities. The macroscopic domain consists of a collection of PUC's (see Fig. 1).

The PUC is defined on a microscopic (local) scale with characteristic length $\ell$. We will use a periodic array of a single sphere (circle) in a cube (square) to obtain effective properties, but such a cell can be easily extended to a more complex structure, without affecting the derivations, unless a clear separation of scales is no longer possible. The ratio of the scales is given by $\varepsilon=\ell / L$. Indeed, a sufficiently small scaling parameter is one of the conditions for separation of scales, which is a necessary condition for applying the homogenization procedure.

The PUC consists of a connected fluid domain $\Omega_{1}$ with an inter-dispersed porous skeleton $\Omega_{\mathrm{m}}$. The two domains added form the total domain of the PUC, i.e., $\Omega=\Omega_{1} \cup \Omega_{\mathrm{m}} \cup \Gamma$. The boundary of the PUC is $\partial \Omega$ and $\Gamma$ denotes the boundary between the fluid region and the grain. The porosity in this system is $\varphi=\left|\Omega_{1}\right| /|\Omega|$. For the 2D computations, we use a square fluid domain with a circular grain in the center (Fig. 1). For the 3D PUC we use the structure shown in Fig. 3 to facilitate comparison with the results in Tardif d'Hamonville et al. (2007). We assume that an adsorbed layer belongs to the grain and has a constant thickness 


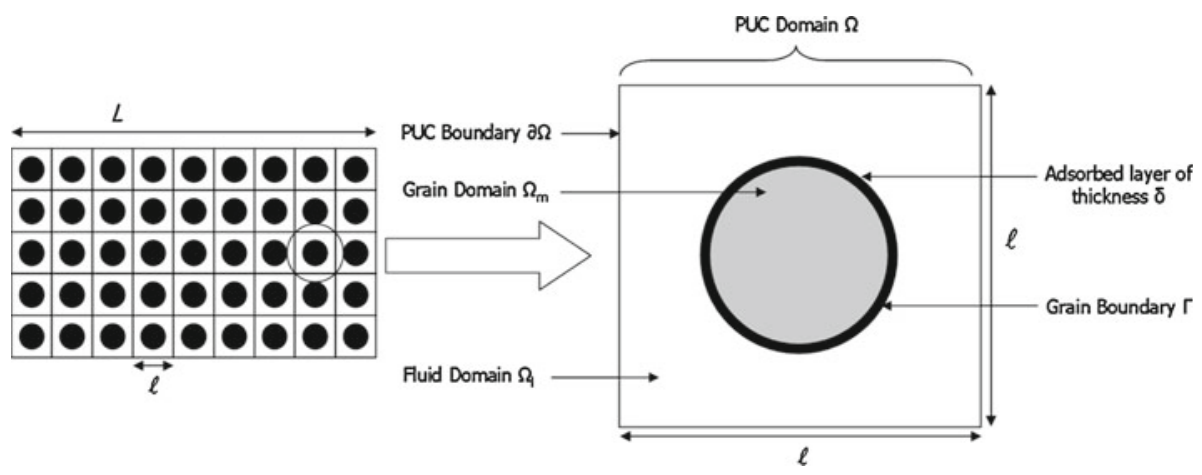

Fig. 1 Periodic lattice on the global scale and PUC on the local scale

$\delta$ independent of the adsorbed concentration. The convection-diffusion equation describing the local scale transport of tracer molecules in the fluid domain of the PUC in terms of the concentration $c$ reads

$$
\frac{\partial c}{\partial t}+\operatorname{div}(\mathbf{v} c)=\operatorname{div}\left(D_{0} \operatorname{grad} c\right),
$$

where $\operatorname{div}(\mathbf{v} c)$ describes the convection and $\operatorname{div}\left(D_{0} \operatorname{grad} c\right)$ describes the diffusion process. Here, $\mathbf{v}$ is the mass averaged velocity of the average velocity of the tracer molecules and water molecules (Bird et al. 1960). The diffusion coefficient in the fluid domain of the PUC is the bulk molecular diffusion coefficient $D_{0}$. The dimensionless small or local scale Peclet number $(\mathrm{Pe})$ gives the ratio between the convective and diffusive transport, i.e.,

$$
P e=\frac{v_{\mathrm{R}} c}{D_{0} \frac{c}{\ell}}=\frac{v_{\mathrm{R}} \ell}{D_{0}},
$$

where the reference velocity $v_{\mathrm{R}}$ is the Darcy injection velocity $u_{\mathrm{inj}}$ divided by the porosity: $v_{\mathrm{R}}=u_{\text {inj }} / \varphi$. In the microscopic setting $u_{\text {inj }}$ is the average flow rate based on the total volume of the unit cell inclusive the grain. The Peclet number is $\left(O\left(\varepsilon^{0}\right)\right)$ with respect to $\varepsilon$ is a representative number at significant distance from wells, where peclet numbers are much higher. We use that $v_{\mathrm{R}}$ is approximately $10^{-5}(\mathrm{~m} / \mathrm{s}), \ell$ is of the order of $10^{-4}(\mathrm{~m})$ and $D_{0}$ for small molecules in water is approximately $10^{-9}\left(\mathrm{~m}^{2} / \mathrm{s}\right)$. Note that this value of the Peclet number can be completely different; for microbes the diffusion coefficient is much smaller $\left(D_{0} \sim 10^{-13}\left(\mathrm{~m}^{2} / \mathrm{s}\right)\right)$, near wells the Darcy velocity may be much higher, whereas in areas with low hydraulic gradients (Bangladesh) the Darcy velocity is much smaller. When flows in the gas phase are considered, the gas diffusion coefficient is of the order of $10^{-5} p_{\text {atm }} / p$ $\left[\mathrm{m}^{2} / \mathrm{s}\right]$, a factor of $10^{4}$ larger than the liquid diffusion coefficients $D_{0}$ at atmospheric pressure $p=p_{\text {atm }}$. Peclet numbers of different orders with respect to $\varepsilon$ may lead to other upscaled equations (Bouddour et al. 1996).

Stokes equation describes the flow in the fluid domain and results in the velocity field $\mathbf{v}=\left(v_{x}, v_{y}, v_{z}\right)$. The Stokes equation for incompressible fluids is

$$
\operatorname{grad} p=\mu \operatorname{div} \operatorname{grad} \mathbf{v}
$$

where $p$ is the pressure and $\mu$ is the viscosity. It describes the velocity field $\mathbf{v}=\left(v_{x}, v_{y}, v_{z}\right)$ in $\Omega_{1}$. Without loss of generality we can ignore gravity effects, by replacing the pressure by the potential $\phi=p+\rho g z$, where $\rho$ is the constant fluid density and $g$ is the acceleration due to gravity. The fluid is assumed to be incompressible and therefore 


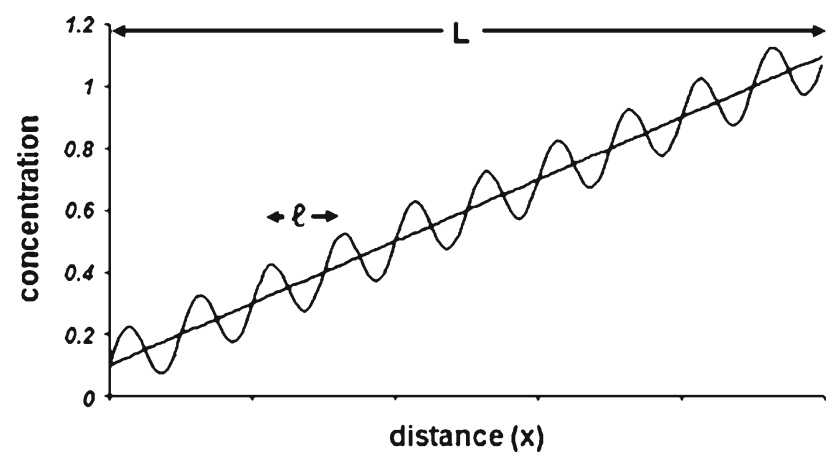

Fig. 2 Plot of the concentration versus distance. The values of $c^{(0)}$ are represented by the straight line and the values of $c^{(1)}$ by the difference between the sinusoidal curve and the straight line (see Eq. 2.7). The length $\ell$ represents the small (pore) scale indicated by sub-index s and the length $L$ represents the large (core) scale as is indicated by the sub-index $b$

$$
\operatorname{div} \mathbf{v}=0 .
$$

We use a no-slip boundary condition at the boundary $\Gamma$ between the solid and the fluid and assume that the grain surface is impermeable, therefore, $\mathbf{v}=0$ at $\Gamma$. Following Auriault and Lewandowska (1996), we can solve Stokes equation independently of the transport equation in the case of tracer flow. We give preference to this approach, which uses a velocity field that is correct to all orders of magnitude, to simplify as much as possible the derivations in the article to enhance its readability, because (i) it is also adopted in Auriault and Lewandowska (1996) and (ii) it leads to the same results. Indeed, it is stated in Auriault and Lewandowska (1996) that "the advective motion is independent of the diffusion and adsorption phenomena, i.e., the coupling term is small with respect to the other ones. Therefore, the classical macroscopic description of the advection (i.e., Darcy law), which has already been presented in earlier contributions (see, for example, Auriault 1991), will be directly used in the analysis". Indeed, the leading order terms in the homogenization procedure lead to Eqs. 2.3 and 2.4. We use Eq. 2.1 as the mass transport equation. At the grain boundary, adsorption of the tracer molecules to the surface takes place. At the boundary the diffusing molecules are adsorbed, i.e.,

$$
(-D \operatorname{grad} c) \cdot \mathbf{n}=\delta\left(\frac{\partial c_{\mathrm{a}}}{\partial t}\right)_{\Gamma} \text { at } \Gamma,
$$

where $\delta$ is the constant thickness of the adsorbed layer and $c_{\mathrm{a}}$ is the equilibrium surface concentration $\left[\mathrm{kg} / \mathrm{m}^{3}\right]$ at the boundary. The relation between the adsorbed concentration $c_{\mathrm{S}}$ (per unit pore volume) and the equilibrium surface concentration $c_{\mathrm{a}}$ reads

$$
c_{\mathrm{s}}=\frac{\delta}{\varphi \ell^{N}} \oint_{\Gamma} c_{\mathrm{a}} \mathrm{d} s,
$$

where $N=2$ for a 2D model and $N=3$ for a 3D model.

The space coordinate at the small scale is indicated by $\mathbf{r}_{\mathrm{s}}$ and the space coordinate at the large scale is indicated by $\mathbf{r}_{\mathrm{b}}$. We split the space derivative terms in the governing equations into a small (local) scale and a large (global) scale contribution (see Fig. 2). We write the concentration $c\left(\mathbf{r}_{\mathrm{s}}, \mathbf{r}_{\mathrm{b}}, t\right)$ and its derivative toward $t$ as formal power series in the scaling parameter $\varepsilon$ 
(a)

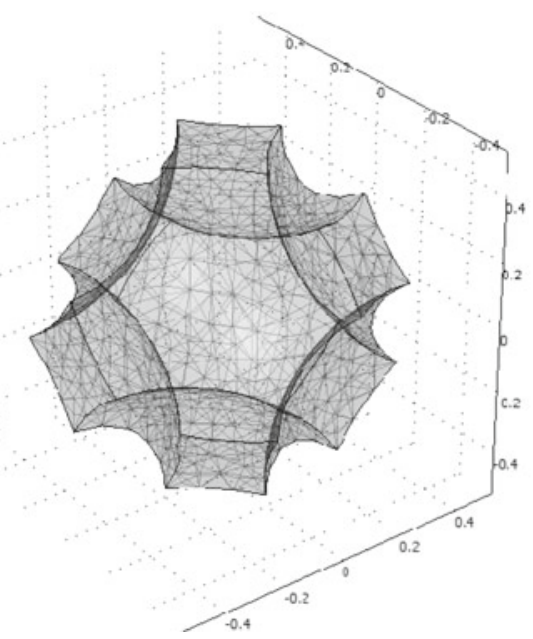

(b)

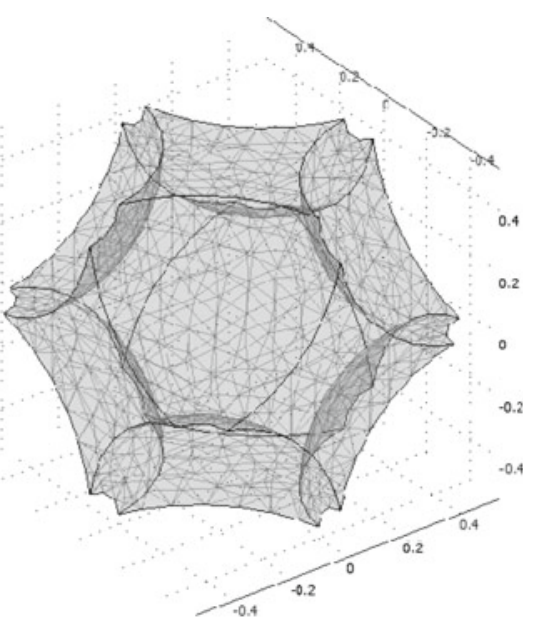

Fig. 3 Part of the PUC (cube) filled with fluids: a finite element mesh with radius of spheres at the corner $a=0.583$ and $\mathbf{b}$ finite element mesh for $a=0.510$ The average $x$-velocity is equal to $1 / \varphi$

$$
\begin{aligned}
c & =c^{(0)}+\varepsilon c^{(1)}+\varepsilon^{2} c^{(2)}+\cdots \\
\frac{\partial c}{\partial t} & =\frac{\partial c^{(0)}}{\partial t}+\varepsilon \frac{\partial c^{(1)}}{\partial t}+\varepsilon^{2} \frac{\partial c^{(2)}}{\partial t}+\cdots
\end{aligned}
$$

Figure 2 illustrates this assumption schematically. Substitution of Eq. 2.7 in Eq. 2.1 and collecting the terms of different orders w.r.t. $\varepsilon$, leads to a set of equations, which can be used to analyze the system. In order to eliminate the local scale dependency of the equations, we average the equation with a certain order of $\varepsilon$ over the PUC domain $\Omega$ and thus obtain the up-scaled equation. For higher orders of $\varepsilon$ more accurate model equations are obtained. This will be illustrated below.

\section{Derivation of the Up-Scaled Equations}

This section describes the homogenization procedure for obtaining upscaled equations from the model equations at the microscale. We define the direction of the pressure gradient as the longitudinal direction. The transverse directions are perpendicular to the direction of the pressure gradient.

\subsection{Boundary and Initial Conditions at the Microscale}

At the grain boundary the velocity is zero; a combination of periodic and semi-periodic boundary conditions are applied on the outer boundaries of the unit cell (see Fig. 1). When we would only be using pure periodic boundary conditions we would write

$$
\begin{aligned}
& c(x=0, y, z, t)=c(x=1, y, z, t) \\
& c(x, y=0, z, t)=c(x, y=1, z, t) \\
& c(x, y, z=0, t)=c(x, y, z=1, t) .
\end{aligned}
$$

However, when we use a combination of semi-periodic boundary conditions at the boundaries of the unit cell in combination with strictly periodic boundary conditions, e.g., we 
would replace $c(x=0, y, z, t)=c(x=1, y, z, t)$ in Eq. 3.1 by the semi-periodic boundary condition

$$
c(x=0, y, z, t)=c(x=1, y, z, t)+1 .
$$

If we were to use only strictly periodic boundary conditions we would obtain trivial solutions, i.e., the concentration $c(x, y, z, t)=$ constant.

We also use semi-periodic boundary conditions for the pressure, e.g., the pressure is periodic in two transverse directions and a given pressure difference is applied between the two faces of the PUC perpendicular to the longitudinal direction.

Tartar shows in the book by Sanchez-Palencia (1980, pp. 368-377) that the homogenized Stokes equation is actually the Darcy equation. The equations on the microscale used in the up-scaling procedure are Eq. 2.1 with boundary condition (2.5) at the grain surface and semi-periodic boundary conditions at $\partial \Omega$.

\subsection{The Non-Dimensional Equations at Two Scales}

The first step in the homogenization process is to write the concentration as $c=c\left(\mathbf{r}_{\mathrm{b}}, \mathbf{r}_{\mathrm{s}}\right)$, i.e., depending on the global coordinate $\mathbf{r}_{\mathrm{b}}$ and the local coordinate $\mathbf{r}_{\mathrm{s}}$, where we omit the indication of the time dependence for convenience. This splits the equations in a small PUC scale term with reference length $\ell$ (see Fig. 2) and a global scale term with reference length $L$, denoted by the subscripts $s$ and $b$, respectively. The introduction of two reference lengths, viz., $L$ and $\ell$ is a convenient way to express that the terms with local differentiation are one order of magnitude larger than the terms involving global differentiation as shown below. The term div becomes $\mathbf{d i v}_{\mathrm{s}}+\mathbf{d i v}_{\mathrm{b}}$. Homogenization assumes that the global contribution $\operatorname{div}_{\mathrm{b}}()$ is one order of magnitude smaller than the local contribution $\operatorname{div}_{\mathrm{s}}()$ with respect to the scaling parameter $\varepsilon=\ell / L \ll 1$. In fact if the two contributions were of the same order of magnitude homogenization cannot be applied (Auriault 1991). The full dimensional equation reads

$$
\begin{gathered}
\frac{\partial c\left(\mathbf{r}_{\mathrm{b}}, \mathbf{r}_{\mathrm{s}}\right)}{\partial t}+\operatorname{div}_{\mathrm{b}}\left(\mathbf{v} c\left(\mathbf{r}_{\mathrm{b}}, \mathbf{r}_{\mathrm{s}}\right)\right)+\operatorname{div}_{\mathrm{s}}(\mathbf{v} c)=D \operatorname{div}_{\mathrm{b}} \operatorname{grad}_{\mathrm{b}} c\left(\mathbf{r}_{\mathrm{b}}, \mathbf{r}_{\mathrm{s}}\right) \\
+D \operatorname{div}_{\mathrm{s}} \operatorname{grad}_{\mathrm{b}} c\left(\mathbf{r}_{\mathrm{b}}, \mathbf{r}_{\mathrm{s}}\right)+D \operatorname{div}_{\mathrm{b}} \operatorname{grad}_{\mathrm{s}} c\left(\mathbf{r}_{\mathrm{b}}, \mathbf{r}_{\mathrm{s}}\right)+D \operatorname{div}_{\mathrm{s}} \operatorname{grad}_{\mathrm{s}} c\left(\mathbf{r}_{\mathrm{b}}, \mathbf{r}_{\mathrm{s}}\right) .
\end{gathered}
$$

We non-dimensionalize Eq. 3.3 by inspection (Shook et al. 1992), i.e., write every dependent and independent variable $Q$ as the product of a dimensionless variable and a reference value $Q=Q_{\mathrm{D}} Q_{\mathrm{R}}$. All reference values $Q_{\mathrm{R}}$ must be related to clearly identifiable quantities in our problem of interest. For the equations used here, $\ell$ is the reference length for the small scale, $L$ the reference length for the large scale, and $v_{\mathrm{R}}=u_{\mathrm{inj}} / \varphi$ is the reference velocity. Here, $u_{\text {inj }}$ is the injection velocity and $\varphi$ denotes the porosity $\varphi=\left|\Omega_{1}\right| /|\Omega|$. As there is no clearly identifiable reference time $t_{\mathrm{R}}$, it must be composed of the other variables, i.e., $t_{\mathrm{R}}=L / v_{\mathrm{R}}$. There are two dimensionless numbers in the convection-diffusion equation, viz., the Peclet number, and the retardation factor. The Peclet number is based on the reference length for the small scale, $P e=v_{\mathrm{R}} \ell / D_{0}$. Thus we find from Eq. 2.1

$$
\begin{gathered}
\frac{\partial c}{\partial t}+\operatorname{div}_{\mathrm{b}}(\mathbf{v} c)+\frac{1}{\varepsilon} \operatorname{div}_{\mathrm{s}}(\mathbf{v} c)=\frac{\varepsilon}{P e} \operatorname{div}_{\mathrm{b}} \operatorname{grad}_{\mathrm{b}} c \\
+\frac{1}{P e} \operatorname{div}_{\mathrm{s}} \operatorname{grad}_{\mathrm{b}} c+\frac{1}{P e} \operatorname{div}_{\mathrm{b}} \operatorname{grad}_{\mathrm{s}} c+\frac{1}{\varepsilon P e} \operatorname{div}_{\mathrm{s}} \operatorname{grad}_{\mathrm{s}} c
\end{gathered}
$$


where we drop the sub-index $D$ e.g., $Q_{\mathrm{D}}$, on the symbols of dimensionless dependent and independent variables to enhance readability.

We slightly deviate from the original approach described in Auriault (2002), Auriault and Adler (1995), Auriault et al. (1992, 2005), Auriault and Lewandowska (1993, 1996, 1997, 2001), and Auriault and Royer (1993a,b). We split the differentiation $\nabla()=\nabla_{\mathrm{b}}()+\nabla_{\mathrm{s}}()$ thus distinguishing between differentiation toward $\mathbf{r}_{\mathrm{s}}$ on the small scale denoted by $\nabla_{\mathrm{s}}\left(c\left(\mathbf{r}_{\mathrm{b}}, \mathbf{r}_{\mathrm{s}}\right)\right)$ and differentiation toward $\mathbf{r}_{\mathrm{b}}$ on the larger scale denoted by $\nabla_{\mathrm{b}}\left(c\left(\mathbf{r}_{\mathrm{b}}, \mathbf{r}_{\mathrm{s}}\right)\right)$. We assume that the term $\nabla_{\mathrm{s}}(.$.$) is one order of magnitude larger than the term \nabla_{\mathrm{b}}(.$.$) . After making the equa-$ tions dimensionless, meaning that now $\nabla()$ means dimensionless differentiation we obtain $\nabla()=\nabla_{\mathrm{b}}()+\nabla_{\mathrm{s}}() / \epsilon$. With this, the description becomes completely equivalent with the conventional approach, but we now understand its physical origin. In the non-dimensionalization, we use $L$ as reference variable for the large scale and $\ell$ as reference variable for the small scale. Using two reference variables for the length helps to remind us to distinguish between local and global terms.

The external boundary conditions at the large scale are a combination of Dirichlet or Neumann conditions. At the small scale, i.e., at the PUC scale (equivalent to the Representative Elementary Volume (REV) scale in averaging), we use periodic boundary conditions as external conditions.

Equivalently, according to Bourgeat and Piatnitski (2004), also Dirichlet or Neumann conditions can be used. Indeed Bourgeat and Piatnitski (2004) show that, if separation of scale is possible, effective properties in random media converge as the scale of the unit cell increases, independent of its boundary conditions (periodic, Dirichlet, or Neumann). However, here we use periodic boundary conditions because the unit cell consists of a symmetry element with a single grain. It is possible to extend the unit cell to several grains until convergence is obtained. As it turns out we get a remarkable result that the unit cell with a single grain is already sufficient to find longitudinal dispersion coefficients that agree with experimental values. We note that at the PUC scale the concentration on one face must differ by a constant from the other face as otherwise all concentrations in the PUC become constant.

For the internal boundary condition Eq. 2.5, the procedure is similar for $\operatorname{grad}()=$ $\operatorname{grad}_{\mathrm{b}}()+\operatorname{grad}_{\mathrm{s}}()$ and we obtain the dimensionless boundary condition

$$
\frac{\varepsilon}{P e}\left(\operatorname{grad}_{\mathrm{b}} c\right) \cdot \mathbf{n}+\frac{1}{P e}\left(\operatorname{grad}_{\mathrm{s}} c\right) \cdot \mathbf{n}=-K\left(\frac{\partial c}{\partial t}\right)_{\Gamma} \quad \text { at } \Gamma,
$$

where we assume that the distribution coefficient

$$
K(c)=\frac{\delta}{L} \frac{\partial c_{\mathrm{a}}}{\partial c}=\varepsilon \frac{\delta}{\ell} \frac{\partial c_{\mathrm{a}}}{\partial c}
$$

is of the order $\varepsilon$. We assume that the adsorbed concentration $c_{\mathrm{a}}(c)$ in the shell of thickness $\delta$ enveloping the grains is in equilibrium with and thus a function of the concentration $c$ in the fluid near the grain surface. It is shown below that the distribution coefficient can be related to the retardation factor $R=1+\frac{K\left(c^{(0)}\right)}{|\Omega| \varphi \varepsilon}|\Gamma|$, where in the unit cell $|\Omega|=1$ and $|\Gamma|$ is the surface area of the grain in the unit cell.

As shown above the reference time $t_{\mathrm{R}}$ can be composed from the reference length $L$ for the large scale divided by the fluid velocity $v_{\mathrm{R}}$ (Shook et al. 1992). Hence, there is no separate dimensionless number connected to the first term of Eq. 3.4. If the interest is in the concentration profile at a distance of the order of $L$ from the origin it follows that $(1+R) \partial c / \partial t$ is of the same order of magnitude as $\operatorname{div}_{\mathrm{b}}(\mathbf{v} c)$. 
3.3 The Flow Equations and Boundary Condition with Terms of the Order of $\varepsilon^{-1}$ and $\varepsilon^{0}$

The terms of the order of $\varepsilon^{-1}$ in the flow equation and $\varepsilon^{0}$ in the boundary condition are the leading order terms. We expand the unknown concentration as a formal power series in the scaling parameter $\varepsilon$ (see Eq. 2.7). All terms $c^{(0)}, c^{(1)}$ etc. are $\Omega$-periodic, i.e., periodic in the PUC. In practice the series is truncated for $\varepsilon^{0}$ or $\varepsilon^{1}$. In other words, $c^{(0)}$ is an approximation of the real behavior of $c$ with an accuracy depending on $\varepsilon$ (Auriault and Royer 1993b). Each of the terms with the same power of $\varepsilon$ are collected to give an equation that provides partial information for solving the total system of equations, because a small change in $\varepsilon$ does not change the upscaling procedure. The terms of lowest order, $\varepsilon^{-1}$, in Eq. 3.4 lead to

$$
\operatorname{div}_{\mathrm{s}}\left(v c^{(0)}\right)=\frac{1}{P e} \operatorname{div}_{\mathrm{s}} \operatorname{grad}_{\mathrm{s}} c^{(0)}
$$

where we use that $\partial c^{(0)} / \partial t$ is of the order of $\varepsilon^{0}$ and can be ignored at the scale $\varepsilon^{-1}$. This assumption implies that our interest is in the development of the concentration profile on the scale $L$. We use that

$$
\operatorname{div}_{\mathrm{s}}\left(\mathbf{v} c^{(0)}\right)=c^{(0)} \operatorname{div}_{\mathrm{S}} \mathbf{v}+\mathbf{v} \cdot \operatorname{grad}_{\mathrm{s}} c^{(0)}=\mathbf{v} \cdot \operatorname{grad}_{\mathrm{s}} c^{(0)},
$$

where $\operatorname{div}_{\mathrm{s}} \mathbf{v}=0$ because of incompressibility. Indeed for fluids at constant density $\operatorname{div}_{\mathrm{s}} \mathbf{v}=$ $\operatorname{div}_{\mathrm{b}} \mathbf{v}=0$. Substitution into Eq. 3.7 leads to

$$
\mathbf{v} \cdot \operatorname{grad}_{\mathrm{s}} c^{(0)}=\frac{1}{P e} \operatorname{div}_{\mathrm{s}}\left(\operatorname{grad}_{\mathrm{s}} c^{(0)}\right) .
$$

The resulting term of order $\varepsilon^{0}$ in boundary condition (3.5) is

$$
\frac{1}{P e}\left(\operatorname{grad} c^{0}\right) \cdot \mathbf{n}=0 \text { at } \Gamma .
$$

The only solution of Eqs. 3.8 and 3.9 that satisfies periodic boundary conditions is that $c^{(0)}$ is constant on a local scale and is therefore independent of the local scale coordinate $\mathbf{r}_{\mathrm{s}}=\left(x_{\mathrm{s}}, y_{\mathrm{s}}, z_{\mathrm{s}}\right)$. Even if $c^{(0)}$ is constant at the local scale it is a non-constant function of the global scale coordinate $c^{(0)}=c^{(0)}\left(\mathbf{r}_{\mathrm{b}}, t\right)=c^{(0)}\left(x_{\mathrm{b}}, y_{\mathrm{b}}, z_{\mathrm{b}}, t\right)$.

\subsection{Equations Derived at Higher Order of $\varepsilon$}

The next step is to analyze the resulting terms of $\varepsilon^{0}$ in Eq. 3.4, i.e.,

$$
\frac{\partial c^{(0)}}{\partial t}+\operatorname{div}_{\mathrm{b}}\left(\mathbf{v} c^{(0)}\right)+\operatorname{div}_{\mathrm{s}}\left(\mathbf{v} c^{(1)}\right)=\frac{1}{P e} \operatorname{div}_{\mathrm{s}}\left(\operatorname{grad}_{\mathrm{b}} c^{(0)}\right)+\frac{1}{P e} \operatorname{div}_{\mathrm{s}}\left(\operatorname{grad}_{\mathrm{s}} c^{(1)}\right),
$$

In many cases of interest it suffices to use a transport equation without the diffusion terms, i.e., the zeroth order transport equation, which can be derived from Eq. 3.10 (see Appendix A).

$$
\frac{\partial c^{(0)}}{\partial t}+\frac{\overline{\mathbf{v}}}{R} \cdot \operatorname{grad}_{\mathrm{b}} c^{(0)}=0,
$$

where the retardation factor

$$
R=1+\frac{K\left(c^{(0)}\right)}{|\Omega| \varphi \varepsilon}|\Gamma|>1 .
$$


Note that $K\left(c^{(0)}\right)$ is constant at the local scale and hence $K\left(c^{(0)}\right)$ does not need to be a linear relation in $c^{(0)}$. Equation 3.11 can be used to eliminate the time derivative from Eq. 3.10. It leads to (see Appendix B) the source, convection-diffusion equation

$$
-\frac{\bar{v}_{x}}{R}+\operatorname{div}_{\mathrm{s}}\left(\mathbf{v}\left(\chi_{x}+x_{\mathrm{s}}\right)\right)=\frac{1}{P e} \operatorname{div}_{\mathrm{s}} \operatorname{grad}_{\mathrm{s}}\left(\chi_{x}+x_{\mathrm{s}}\right)
$$

where $\chi_{x}$ is the $x$-component of a vector $\vec{\chi}$ that describes the first order concentration correction $c^{(1)}=\vec{\chi} \cdot \operatorname{grad}_{\mathrm{b}} \mathbf{c}^{(\mathbf{0})}$. When we are interested in the longitudinal dispersion coefficient, $\operatorname{grad}_{\mathrm{b}} c^{(0)}$ is applied in the same direction as the overall pressure gradient, whereas if we are interested in the transverse dispersion coefficient, $\operatorname{grad}_{\mathrm{b}} c^{(0)}$ is applied perpendicular to the overall pressure gradient. The velocity $\mathbf{v}$ is the mass averaged velocity on the microscale (see Sect. 2).

In the same way, we collect the terms of order $\varepsilon^{1}$ in Eq. 3.5,

$$
\frac{1}{P e}\left(\operatorname{grad}_{\mathrm{b}} c^{(0)}\right) \cdot \mathbf{n}+\frac{1}{P e}\left(\operatorname{grad}_{\mathrm{s}} c^{(1)}\right) \cdot \mathbf{n}=-\frac{K\left(c^{(0)}\right)}{\varepsilon}\left(\frac{\partial c^{(0)}}{\partial t}\right) \Gamma \quad \text { at } \Gamma,
$$

where $K\left(c^{(0)}\right) / \varepsilon$ is of order $\varepsilon^{0}$.

Substitution of $c^{(1)}=\vec{\chi} \cdot \operatorname{grad}_{\mathrm{b}} c^{(0)}$ in the boundary condition Eq. 3.14, combining with Eq. 3.11 and looking at the case $\operatorname{grad}_{\mathrm{b}} c^{(0)}=-\mathbf{e}_{x}$ leads with the relation between $K\left(c^{(0)}\right)$ and the retardation factor $R$ defined in Eq. 3.12, i.e., $\frac{K\left(c^{(0)}\right)}{|\Omega| \varphi \varepsilon}|\Gamma|=R-1$ to (see Appendix B)

$$
\frac{1}{P e}\left(\operatorname{grad}_{\mathrm{s}}\left(\chi_{x}+x_{\mathrm{s}}\right)\right) \cdot \mathbf{n}=\frac{K\left(c^{(0)}\right)}{\varepsilon} \frac{\bar{v}_{x}}{R}=\frac{(R-1)|\Omega|}{R|\Gamma|} \varphi \bar{v}_{x} \quad \text { at } \Gamma .
$$

Note that $|\Gamma|$ is the dimensionless surface area of the grain, i.e., in the PUC with a volume equal to one.

We can calculate $c^{(1)}$ from Eqs. 3.13 and 3.15 with the help of a numerical method. In this study, we use the finite element software package COMSOL for this purpose to solve for $\chi_{x}$.

Retaining the terms up to order $\varepsilon^{1}$ leads to the complete higher order convection-diffusion equation (see Appendix C), i.e.,

$$
\left\langle\frac{\partial c^{(1)}}{\partial t}\right\rangle+\operatorname{div}_{\mathrm{b}}\left\langle\mathbf{v} c^{(1)}\right\rangle=\frac{1}{P e} \operatorname{div}_{\mathrm{b}}\left(\left\langle\operatorname{grad}_{\mathrm{b}} c^{(0)}\right\rangle+\left\langle\operatorname{grad}_{\mathrm{s}} c^{(1)}\right\rangle\right)-\int_{\Gamma} \frac{K\left(c^{(0)}\right)}{|\Omega| \varepsilon}\left(\frac{\partial c^{(1)}}{\partial t}\right)_{\Gamma} \mathrm{d} s .
$$

where $\langle Q\rangle$ means that $Q$ is averaged over the PUC.

\section{Derivation of the Dispersion Coefficients}

\subsection{The Up-Scaled Dimensionless Equation}

Since, we now have an expression for the global concentration $\frac{\partial c^{(0)}}{\partial t}$ from Eq. 3.10 and an expression for the first order correction $\frac{\partial c^{(1)}}{\partial t}$ from Eq. 3.16, we can find an up-scaled equation that includes diffusion. 
By adding the product of Eq. 3.16 and $\varepsilon$ to Eq. 3.10 and by substitution of $c^{(1)}=\vec{\chi}$. $\operatorname{grad}_{\mathrm{b}} c^{(0)}$, we obtain the dimensionless up-scaled convection-diffusion equation

$$
\begin{aligned}
& \left\langle\frac{\partial c}{\partial t}\right\rangle+\operatorname{div}_{\mathrm{b}}\left\langle\mathbf{v} c^{(0)}\right\rangle=-\varepsilon \operatorname{div}_{\mathrm{b}}\langle(\mathbf{v} \otimes \vec{\chi})\rangle \cdot \operatorname{grad}_{\mathrm{b}} c^{(0)} \\
& +\frac{\varepsilon}{P e} \operatorname{div}_{\mathrm{b}}\left\langle\left(\mathbf{I}+\operatorname{grad}_{\mathrm{s}} \otimes \vec{\chi}\right)\right\rangle \cdot \operatorname{grad}_{\mathrm{b}} c^{(0)}-\frac{1}{|\Omega| \varepsilon} \int_{\Gamma} K\left(c^{(0)}\right)\left(\frac{\partial c}{\partial t}\right) \mathrm{d} s,
\end{aligned}
$$

where we use the notation $c=c^{(0)}+\varepsilon c^{(1)}$.

Elaboration of some averages leads to

$$
\begin{aligned}
\varphi \frac{\partial c^{0}}{\partial t}+\operatorname{div}_{\mathrm{b}}\left(\overline{\mathbf{u}} c^{0}\right)= & \varepsilon \operatorname{div}_{\mathrm{b}}\left(-\langle(\mathbf{v} \otimes \vec{\chi})\rangle+\frac{1}{P e}\left\langle\left(\mathbf{I}+\operatorname{grad}_{\mathrm{s}} \otimes \vec{\chi}\right)\right\rangle\right) \cdot \operatorname{grad}_{\mathrm{b}} c^{(0)} \\
& -\frac{1}{|\Omega| \varepsilon} \int_{\Gamma} K\left(c^{(0)}\right)\left(\frac{\partial c}{\partial t}\right)_{\Gamma} \mathrm{d} s,
\end{aligned}
$$

where $\left\langle\frac{\partial c}{\partial t}\right\rangle=\varphi \frac{\partial c^{(0)}}{\partial t}$ because $\varepsilon\left\langle\frac{\partial c^{(1)}}{\partial t}\right\rangle=0$ as the average of the higher order concentration terms over the PUC is zero. We can rewrite the average interstitial velocity $\langle\mathbf{v}\rangle$ over the PUC using the average Darcy velocity: $\overline{\mathbf{u}}=\frac{1}{|\Omega|} \int_{\Omega_{1}} \mathbf{v} \mathrm{d} \mathbf{r}_{\mathrm{s}}=\varphi \overline{\mathbf{v}}$. We only consider cases where the main pressure gradient is applied perpendicular to one of the faces, with the normal in the $x, y$, or $z$-direction, of the cubic unit cell. By applying the global concentration gradient in the same direction as the pressure gradient we obtain the longitudinal dispersion coefficient and when we apply the global concentration gradient perpendicular to the pressure gradient we obtain the transverse dispersion coefficient. By virtue of symmetry the average velocity in the direction perpendicular to the applied pressure gradient is zero. The average velocity $v_{x}$ over the west-boundary of the unit cell is the Darcy velocity (volumetric flux). The flux is constant over any cross-section $x=c$ in the unit cell and hence the integral of the Stokes velocity in the unit cell is equal to the Darcy velocity.

\subsection{Hydrodynamic Dispersion and Effective Diffusion Coefficients}

We recall that the dimensionless variables, $Q_{\mathrm{D}}=Q / Q_{\mathrm{R}}$, are expressed as the ratio between the full dimensional variable $(Q)$ and the reference value $\left(Q_{\mathrm{R}}\right)$. On the microscale the reference length is $\ell$, where as on the macroscale it is $L$, with $\varepsilon=\ell / L$. For the reference time, we used $t_{\mathrm{R}}=L / v_{\mathrm{R}}$, where $v_{\mathrm{R}}=u_{\mathrm{inj}} / \varphi$ is the reference velocity. We also recall that $P e=v_{\mathrm{R}} \ell / D_{0}$ and $K=\frac{\delta}{L} \frac{\partial c_{\mathrm{a}}}{\partial c}$. We dropped the sub-index $D$ in the paper after we converted to dimensionless equations. Therefore, we replace $Q \rightarrow Q / Q_{\mathrm{R}}$. Finally, we recall that $\overline{\mathbf{u}}=\varphi \overline{\mathbf{v}}$. Even if the derivation is straightforward we prefer to mention the intermediate step, i.e., in fully dimensional form we obtain for Eq. 4.2

$$
\begin{aligned}
\varphi t_{\mathrm{R}} \frac{\partial c^{0}}{\partial t}+\frac{L}{v_{\mathrm{R}}} \operatorname{div}_{\mathrm{b}}\left(\overline{\mathbf{u}} c^{0}\right)= & \varepsilon L^{2} \operatorname{div}_{\mathrm{b}}\left(\left(-\langle(\mathbf{v} \otimes \vec{\chi})\rangle+\frac{1}{P e}\left\langle\left(\mathbf{I}+\operatorname{grad}_{\mathrm{s}} \otimes \vec{\chi}\right)\right\rangle\right) \cdot \operatorname{grad}_{\mathrm{b}} c^{(0)}\right) \\
& -\frac{\ell^{3} t_{\mathrm{R}}}{|V| \ell^{2} \varepsilon} \frac{\delta}{L}\left(\frac{\partial c_{\mathrm{a}}}{\partial c}\right)_{c^{(0)}}\left(\frac{\partial c^{(0)}}{\partial t}\right) \int_{\Gamma} \mathrm{d} s,
\end{aligned}
$$

where we assumed that the average of the concentration $c^{(1)}$ over the grain surface is zero. The terms in averaging brackets are still in their dimensionless form. We note that the integral over the surface area of the particle divided by the volume of the unit cell is the specific surface $S=\frac{1}{V} \int_{\Gamma} \mathrm{d} s$. We obtain after division by $t_{\mathrm{R}}$ 


$$
\begin{aligned}
& \varphi \frac{\partial c^{0}}{\partial t}+\operatorname{div}_{\mathrm{b}}\left(\overline{\mathbf{u}} c^{0}\right)=-S \delta\left(\frac{\partial c_{\mathrm{a}}}{\partial c}\right)_{c^{(0)}}\left(\frac{\partial c^{(0)}}{\partial t}\right)_{\Gamma} \\
& +\frac{\varepsilon L^{2}}{t_{\mathrm{R}}} \operatorname{div}_{\mathrm{b}}\left(\left(-\langle(\mathbf{v} \otimes \vec{\chi})\rangle+\frac{1}{P e}\left\langle\left(\mathbf{I}+\operatorname{grad}_{\mathrm{s}} \otimes \vec{\chi}\right)\right\rangle\right) \cdot \operatorname{grad}_{\mathrm{b}} c^{(0)}\right) .
\end{aligned}
$$

This equation is conventionally written in hydrology as

$$
\varphi R \frac{\partial c^{0}}{\partial t}+\operatorname{div}_{\mathrm{b}}\left(\overline{\mathbf{u}} c^{0}\right)=\operatorname{div}_{\mathrm{b}}\left(\left(\varphi \mathbf{D}_{\mathrm{d}}+\varphi \mathbf{D}_{\mathrm{m}}\right) \cdot \operatorname{grad}_{\mathrm{b}} c^{(0)}\right),
$$

where $\mathbf{D}_{\mathrm{d}}$ denotes the hydrodynamic dispersion coefficient and $\mathbf{D}_{\mathrm{m}}$ denotes the contribution of the molecular diffusion coefficient modified by the tortuosity tensor $\operatorname{grad}_{\mathrm{s}} \otimes \vec{\chi}$. We use that $S \delta\left(\frac{\partial c_{\mathrm{a}}}{\partial c}\right)_{c^{(0)}}=\varphi(R-1)$, the product of the porosity and the retardation factor minus one.

We note that $\frac{\varepsilon L^{2}}{t_{\mathrm{R}}}=\ell v_{\mathrm{R}}=D_{0} P e$. Comparison of Eqs. 4.3 and 4.4 leads to the expressions

$$
\mathbf{D}_{\mathrm{d}}=-\frac{D_{0} P e}{\varphi} \frac{1}{|\Omega|} \int_{\Omega_{1}} \mathbf{v} \otimes \vec{\chi} \mathrm{d} \mathbf{r}_{\mathrm{s}}
$$

and

$$
\mathbf{D}_{\mathrm{m}}=\frac{D_{0}}{\varphi}\left(\varphi \mathbf{I}+\frac{\varphi}{\left|\Omega_{1}\right|} \int_{\Omega_{1}} \operatorname{grad}_{\mathrm{s}} \otimes \vec{\chi} \mathrm{d} \mathbf{r}_{\mathrm{s}}\right),
$$

where we keep $\mathbf{v} \otimes \vec{\chi}$ and $\operatorname{grad}_{\mathrm{s}} \otimes \vec{\chi}$ still in their dimensionless form as they are computed from Eqs. 3.13 and 3.15, which are kept in the dimensionless setting. For the computation of the dispersion coefficients, below, we have chosen $x$ as the direction of global or large scale fluid flow. In the equations, $\chi_{x}$ is strictly periodic (see Eq. 3.1). Without adsorption the right side of Eq. 3.15 is zero as $R=1$. When including adsorption the retardation factor $R>1$. The equations obtained in Tardif d'Hamonville et al. (2007) are similar. However, the engineering community, for historical reasons, does not include the porosity $(\varphi)$ effect in the dispersion coefficient and therefore Eqs. 4.5 and 4.6 use a division by $\varphi$ in contrast to Tardif d'Hamonville et al. (2007). Note that $\left|\Omega_{1}\right|=\varphi$. We use the Peclet number $(P e)$ in Eq. 4.5 because in our case the velocity $\mathbf{v}$ is still dimensionless, and it is convenient to calculate the integral directly from the dimensionless numerical results.

Note also that certain symmetry conditions are required if $\operatorname{grad}_{\mathrm{s}} \otimes \vec{\chi}$ represents the unit tensor divided by a constant tortuosity factor. Without these conditions the tortuosity factor can become direction dependent. The longitudinal hydrodynamic dispersion coefficient can be computed from

$$
D_{x x, \mathrm{~d}}=-\frac{D_{0} P e}{\varphi} \frac{1}{|\Omega|} \int_{\Omega_{1}} v_{x} \chi_{x} \mathrm{~d} \mathbf{r}_{\mathrm{s}},
$$

and for $x x$ component of the molecular diffusion tensor reads

$$
D_{x x, \mathrm{~m}}=D_{0}\left(1+\frac{1}{\left|\Omega_{1}\right|} \int_{\Omega_{1}} \frac{\partial \chi_{x}}{\partial x} \mathrm{~d} \mathbf{r}_{\mathrm{s}}\right) .
$$


All off-diagonal elements of the dispersion tensor will be zero, because $\chi_{x} v_{y}$ is a product of an even function $\chi_{x}$ of $y$ and an uneven function $v_{y}$ of $y$, which upon integration will lead to zero. In the same way, $\partial \chi_{x} / \partial y$ is an uneven function of $y$ and hence the volume integral is zero.

To obtain $\chi_{y}$, we need to solve

$$
\operatorname{div}_{\mathrm{s}}\left(\mathbf{v}\left(\chi_{y}+y_{\mathrm{s}}\right)\right)=\frac{1}{P e} \operatorname{div}_{\mathrm{s}} \operatorname{grad}_{\mathrm{s}}\left(\chi_{y}+y_{\mathrm{s}}\right)
$$

with analogous periodic boundary conditions (like in Eq. 3.2). The grain boundary condition reads, irrespective of whether we use $R>1$,

$$
\frac{1}{P e}\left(\operatorname{grad}_{\mathrm{s}}\left(\chi_{y}+y_{\mathrm{s}}\right)\right) \cdot \mathbf{n}=0 \quad \text { at } \Gamma,
$$

because $\bar{v}_{y}=0$.

Hence, we obtain for the transverse dispersion coefficients

$$
D_{y y, \mathrm{~d}}=-\frac{D_{0} P e}{\varphi} \frac{1}{|\Omega|} \int_{\Omega_{1}} v_{y} \chi_{y} \mathrm{~d} \mathbf{r}_{\mathrm{s}},
$$

and for $y y$ component of the molecular diffusion tensor

$$
D_{y y, \mathrm{~m}}=D_{0}\left(1+\frac{1}{\left|\Omega_{1}\right|} \int_{\Omega_{1}} \frac{\partial \chi_{y}}{\partial y} \mathrm{~d} \mathbf{r}_{\mathrm{s}}\right) .
$$

\section{Numerical Calculation of the Dispersion Coefficient}

In order to calculate the fully dimensional hydrodynamic dispersion and effective diffusion coefficients we need to compute the first order concentration correction $\left(\chi_{x}\right)$ from Eq. 3.13 for the longitudinal dispersion coefficient and the first order correction $\left(\chi_{y}\right)$ from Eq. 4.9 for the transverse dispersion coefficient. We solve the problem both in 2D and 3D using a Finite Element Method software package, COMSOL Multiphysics, but certainly equivalent packages, e.g., FENICS can also be used. COMSOL, formerly FEMLAB is a software package that can solve various coupled engineering and physics problems, e.g., here a combination of Stokes and the convection-diffusion equation. The benefit of using COMSOL with respect to the vorticity approach (Bruining and Darwish 2006) is that it is capable of solving the problem in three dimensions. COMSOL also allows to compute the volume integrals in Eqs. 4.7, $4.8,4.11$, and 4.12. Finite element commercial software makes it easy to do the numerical calculations, even if some background knowledge is required for a proper validation of the results. For the $2 \mathrm{D}$ example, we use a simple square array of cylinders, i.e., the PUC is a circle in a square such that the porosity $\varphi=0.37$. For reasons of easy comparison with Tardif d'Hamonville et al. (2007) we have defined the geometry in Fig. 3 for the PUC in 3D. To obtain the unit cells in Fig. 3, we start with a unit cube. In each of the eight corners of the cube, spheres, representing the grains, with radii of 0.583 or 0.510 have been drawn corresponding to porosities of 0.242 and 0.446 , respectively. The parts of the sphere that fall outside the unit cube are discarded, whereas the parts inside the cube constitute the grains. The overlapping parts of the spheres inside the cube belong to the porous skeleton.

The steady state incompressible Stokes equation $\operatorname{grad} p=\mu$ div $\operatorname{grad} \mathbf{v}$ was implemented by setting the appropriate parameters equal to zero, i.e., discarding the inertia term. The internal flow boundaries are set to a no-slip condition, i.e., zero velocity at the grain surface. 
Symmetry conditions at the cube faces parallel to the main flow direction are equivalent to periodic boundary conditions if the sphere is located at the center of the PUC. Numerical comparisons show that semi-periodic boundary conditions for symmetric PUC's can be set by choosing a constant pressure difference between the corresponding points at the cube faces perpendicular to the main flow direction. The pressure difference between corresponding points was chosen such that the average dimensionless longitudinal velocity $\bar{v}_{x}$ is equal to $1 / \varphi$. This choice of setting $\bar{v}_{x}=1 / \varphi$ reduces the number of calculations since we use this velocity as a factor in the source term of Eq. 3.13. This is accomplished as follows: in the dimensionless calculation in the unit cell with side $\xi=1$, a pressure difference between corresponding points is applied such that the volume integral of the $x$-Darcy-velocity in the flowing part is equal to one, and the choice of the Peclet number is made by varying the molecular diffusion coefficient. Here, taking the volume integral equal to one, means that the average Darcy velocity $\bar{u}$ or specific discharge is one and thus $\bar{v}_{x}$ is equal to $1 / \varphi$. However, it is possible to use any value for $\bar{u}$ as long as the Peclet number $P e=\bar{u} \xi /\left(\varphi D_{0}\right)$. In the literature (Bear 1972), the Peclet number is usually defined as $\bar{v} d_{\mathrm{p}} / D_{0}$, where the interstitial velocity $\bar{v}=\bar{u} / \varphi$ and $d_{\mathrm{p}}$ is the grain diameter. For our choice of inscribed radii ( $a=0.51$ or $a=0.583$ ) this only leads to a minor difference.

Indeed, the advantage of this procedure is that the solution for the velocity field does not change for computations with various Peclet numbers. We can solve the cell equation Eq. 3.13 for the longitudinal dispersion coefficient and Eq. 4.9 for the transverse dispersion coefficient with the stored velocity field as input. We also use boundary condition (3.15). It turns out to be advantageous to use the diffusion equations, Eqs. 3.13 and 4.9 in the transient mode: $\partial c / \partial t+\mathbf{v} \cdot \operatorname{grad} c=\operatorname{div}(D \operatorname{grad} c)+S_{\mathrm{R}}$, where we use $c=\chi_{x}+x_{\mathrm{s}}$ or $c=\chi_{y}+y_{\mathrm{S}}$ for the longitudinal and transverse coefficients, respectively. The source term is only nonzero for longitudinal dispersion, i.e., $S_{\mathrm{R}}=\bar{v}_{x} / R$ while various values of $D=\frac{1}{P e}$ are used in Eqs. 3.13 or 4.9. Here, $x_{\mathrm{s}}\left(y_{\mathrm{s}}\right)$ is the $x$-coordinate ( $y$-coordinate) in the PUC. For long times the solution converges to the solution of the stationary reaction-diffusion-convection equation, i.e., $\mathbf{v} \cdot \operatorname{grad} c=\operatorname{div}(D \operatorname{grad} c)+S_{\mathrm{R}}$. For the calculation of the longitudinal dispersion coefficient, we use semi-periodic boundary conditions in the longitudinal direction, i.e., $c\left(x_{\mathrm{s}}=1, y_{\mathrm{s}}\right)+1=c\left(x_{\mathrm{s}}=0, y_{\mathrm{s}}\right)$ and periodic boundary conditions in the transverse direction, i.e., $c\left(x_{\mathrm{s}}, y_{\mathrm{s}}=1\right)=c\left(x_{\mathrm{s}}, y_{\mathrm{s}}=0\right)$. For reasons of symmetry the latter can also be replaced by the no flow/symmetry boundary condition.

To implement semi-periodic boundary conditions in COMSOL (see Eq. 3.2), it appears to be necessary to choose the appropriate Neumann precondition at the inflow and outflow boundary to avoid discontinuous jumps of the concentration derivative at the boundary; in this case, we implement the convective flux condition $(-D \operatorname{grad} c) \cdot \mathbf{n}=0$ to ensure that the diffusive flux or the concentration gradient is also periodic. This aspect was not incorporated in the COMSOL manual. The remaining external boundaries and the internal boundaries have insulation symmetry boundary conditions, $(-D \operatorname{grad} c+c \mathbf{v}) \cdot \mathbf{n}=0$. The transient solution for the convection-diffusion equation reaches a steady state solution if the source term $S_{\mathrm{R}}=-\bar{v}_{x} / R$ counterbalances the difference between the convective flux entering and leaving the PUC.

To compute the transverse dispersion coefficient, we use $c\left(x_{\mathrm{s}}, y_{\mathrm{s}}=0, z_{\mathrm{s}}, t\right)=c\left(x_{\mathrm{s}}, y_{\mathrm{s}}=\right.$ $\left.1, z_{\mathrm{s}}, t\right)+1$, and strictly periodic boundary conditions in the flow direction $x_{\mathrm{s}}$ and the direction $z_{\mathrm{s}}$. Note that now we cannot replace the boundary condition in the $x$-direction (fluid flow direction) by a no flow/symmetry boundary condition. COMSOL can solve the Stokes equation as well as the convection-diffusion equation in their conservative form. A Multigrid preconditioner presolves the linear set of equations before COMSOL applies the Generalized Minimal Residual Method (GMRES). 


\section{Results}

Figure 4 presents the 2D results of the computations in terms of the ratio of dispersion coefficients $D$ and the molecular diffusion coefficient $D_{0}$ for various Peclet numbers and for a simple square arrays of cylinders with a porosity $\varphi=0.37$ (see Fig. 1) in the absence of adsorption. The Peclet number is defined as $P e=\bar{v} \ell / D_{0}$, where $v$ is the average interstitial velocity (the Darcy velocity or specific discharge divided by $\varphi$ ), the length of the unit cell and $D_{0}$ the molecular diffusion coefficient.

Figure 4 also compares our results with experimental (Bear 1972) and numerical data cited in Table IV of Edwards et al. (1991). The comparisons involve the dispersion in a 2D periodic medium of circles inside squares for a case with porosity $\varphi=0.37$. At the smallest resolution, we used 250 triangular elements and at the highest resolution we used 4,000 triangular elements, with no significant change in the results. Edwards et al. (1991) used 400 nine-node elements. As it turns out Edwards solves exactly the same cell equation (3.13), but state that they derive subsequently the dispersion coefficient from an equation derived by Brenner (1980) (based on a moment analysis)

$$
\mathbf{D}_{\mathrm{m}}+\mathbf{D}_{\mathrm{d}}=\frac{1}{\varphi|\Omega|} \int_{\Omega_{1}} \nabla \vec{\chi} \otimes \nabla \vec{\chi} \mathrm{d} \mathbf{r}_{\mathrm{s}} .
$$

The result of using Eq. 6.1 is shown in Fig. 4 as the thin drawn line below the other data. It only gives good results for very small Peclet numbers. However, the values in Table IV of Edwards et al. (1991) are exactly reproduced for low Peclet numbers if we use Eqs. 4.7 and 4.8 instead of Eq. 6.1. Buyuktas and Wallender (2004) also use Eqs. 3.13, 4.7, and 4.8 to obtain the dispersion coefficient in the same way as in this article. The data from Eidsath et al. (1983) also quoted in Edwards et al. (1991) disagree both with our calculations and the data of Edwards. However, Eidsath used a 36 element mesh. At higher Peclet numbers, the computed data by Edwards are higher than the experimental data and our computed results. We are not able to find a reason for this discrepancy.

Figure 5 shows the 3D results. The 3D simulation with the corner spheres of radii $a=$ 0.510 (0.583) was carried out with 5,832 (3128) mesh points, with 27,420 (14495) tetrahedral

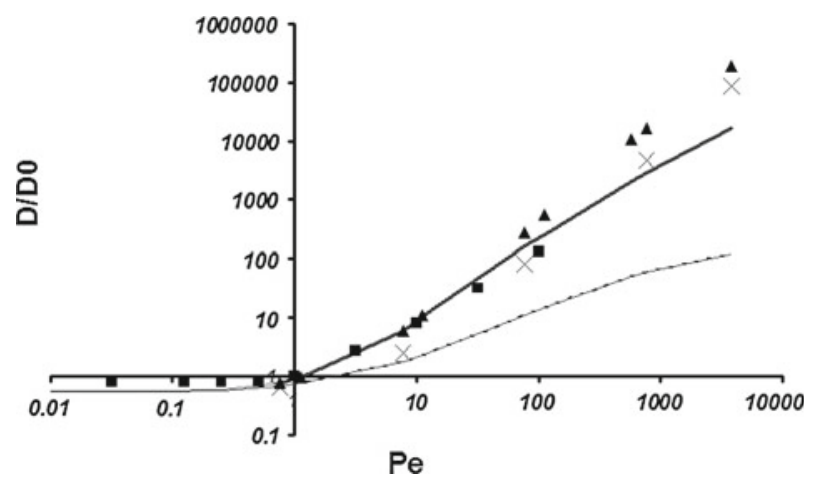

Fig. 4 Comparison of the computed hydrodynamic dispersion coefficients without adsorption (drawn line) for a 2-D model with experimental and numerical data of other authors. The squares are the experimental data (Bear 1972), the crosses are the data from Eidsath et al. (1983) whereas the triangles are data from Edwards et al. (1991). The drawn curve is computed in this work for simple square arrays of cylinders with $\varphi=0.37$. The thin drawn line below the other data uses the cell average of $\left\langle c_{x} c_{x}\right\rangle$ (see Eq. 6.1) to estimate the dispersion coefficient 


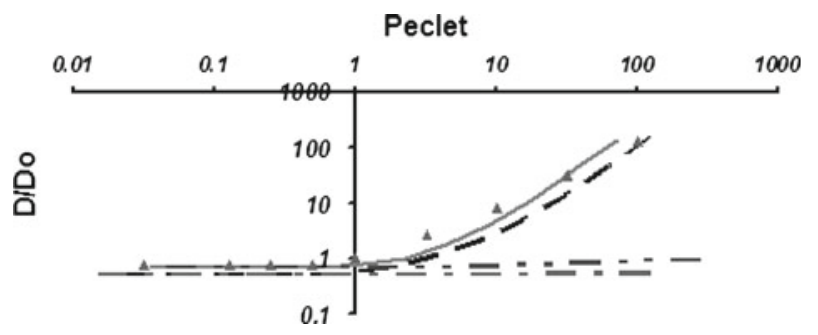

Fig. 5 Longitudinal (upper curves) and transverse (lower curves) dispersion without adsorption divided by molecular diffusion versus Peclet number. The Peclet number (see Eq. 2.2) is based on the interstitial velocity $v=u / \varphi$. The characteristic dimension is the size of the unit cell. Dashed (dashed-dot-dot) line has a unit cell as Fig. 3a and the drawn (dashed-dot) line as Fig. 3b. The triangles denote experimental points (Bear 1972)

Lagrangian quadratic elements. COMSOL uses shlag $\left(2, \mathrm{c}^{\prime}\right)$ shape functions with integration order 4 and constraint order 2. A simulation with 1,731 (955) mesh points and 7,746 (4068) elements gave results that deviated at most $0.133 \%$ (0.288\%). At low Peclet numbers the longitudinal and transverse dispersion coefficient are dominated by the molecular diffusion and thanks to the definition used by engineers they do not depend on the porosity. For low Peclet numbers transverse and longitudinal dispersion are equal. For the configuration in Fig. 3a, b $D / D_{0}$ assumes values of $0.51(0.69)$. The measured value is $D \sim 0.7 D_{0}$, is only obtained for the configuration shown in Fig. 3b. This configuration has a porosity value $\varphi=0.446$, close to many laboratory tests $(\varphi=0.35-0.45)$. The configuration of Fig. 3a has a porosity value of 0.242 , and here we find $D \sim 0.5 D_{0}$ for low Peclet numbers. At high Peclet numbers the longitudinal $D / D_{0}$ values increase faster than proportional to the Peclet number. Deviations between the theoretical results for the longitudinal dispersion coefficients for the configurations of Fig. 3a, b are well within the range of experimentally determined values (Dullien 1992).

It should be noted that experiments to obtain these data are not trivial due to the low values of the dispersion coefficients. Many experimental data that show large values can be incorrect due to stream line splitting at the entrance and production point, i.e., if special fluid distributors at the injection and production point were not used. Small entrapped air bubbles also can cause an apparent increase of the dispersion coefficient. Finally, also the sand pack must be homogeneous, which requires special experimental preparation techniques (Wygal 1963). Some of the experimental values in Bear (1972) are given here as the triangles in Fig. 5.

The transverse dispersion coefficients remain almost equal to the value at low Peclet values. For the configuration in Fig. $3 \mathrm{a}, \mathrm{b}$ the transverse dispersion coefficient divided by increases from $0.51(0.69)$ at low Peclet numbers to 0.59 (1.0) at high Peclet numbers. It also increases slower than proportional to the Peclet number.

There are excellent overview articles for experimental data (Delgado 2006, 2007) of longitudinal and transverse dispersion (Wronski and Molga 1987). These studies mention effects of particle size and particle shape distribution. Non-uniform particle size distributions decrease the value of the longitudinal dispersion. To study size distribution effects with the PUCs used in this study is well possible, but considered outside the scope of the article.

We also compared our results with the results obtained by Tardif d'Hamonville et al. (2007) and found good agreement with their results, taking into account that in Tardif d'Hamonville et al. (2007) the division by the porosity $\varphi$, commonly used by engineers, is not included. In Tardif d'Hamonville et al. (2007), only results for low Peclet numbers are shown. Computed transverse dispersion coefficients are much smaller than experimental values (Delgado 2007). 


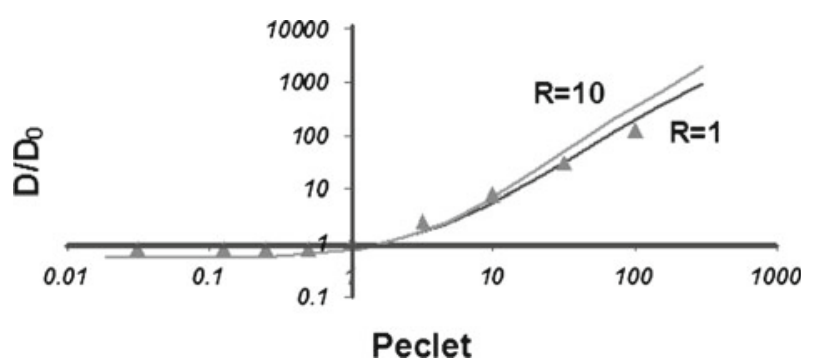

Fig. 6 The effect of adsorption on the dispersion coefficient. With adsorption, i.e., the retardation factor $R=10$, the longitudinal dispersion coefficient is higher than the dispersion coefficient with retardation factor $R=1$, i.e., without adsorption

Possibly a statistical-oriented approach is necessary to obtain more realistic values of the transverse dispersion coefficient (Fannjiang and Papanicolaou 1996). For example, the transverse dispersion coefficients in a staggered array (Edwards et al. 1991) are larger than for the situation of a single sphere in a square. However, the values are still much smaller than experimental values (Delgado 2007). It can be expected that better results can be obtained for more complicated unit cells, but such a cell must have isotropic properties as otherwise longitudinal dispersion mixes in the transverse dispersion.

Figure 6 shows the effect of adsorption on the dispersion coefficient. Such an effect of adsorption can be expected as the movement of solute in the direction of flow is retarded near the grains and not affected far away from the grains, leading to a spreading of solute. The effect of adsorption on dispersion is quantified in the boundary condition for $\vec{\chi}$ in Eq. 3.15. Non-linear adsorption can have a substantial effect on the spreading of a concentration profile (Rhee et al. 2001) on the macroscale, but as we see in Fig. 6, the effect on the longitudinal dispersion coefficient on the local scale is very small. As we discussed in Sect. 4.2, the effect on transverse dispersion is zero.

We end with a few words about the practical relevance of the results in this article. The first important aspect is that homogenization shows whether the proposed up-scaled equation can be used for the interpretation of laboratory results. In periodic media modeling these order of magnitude considerations are ignored. The main condition is that the Peclet number on the PUC scale is of the order of unity. For iron ions with molecular diffusion coefficients in water of the order of $10^{-9}\left[\mathrm{~m}^{2} / \mathrm{s}\right]$ this is clearly the case. For microbes with a much lower diffusion coefficient such an assumption is not correct and this may have a consequence for the up-scaled convection-diffusion equation for microbes. The second application is that it is in principle possible to derive the transport coefficients. A periodic array of single spheres in a cube appears to be sufficient to estimate longitudinal dispersion coefficients. A possible shortcoming of such a simple unit cell manifests itself in the underestimate of the transverse dispersion coefficient. Whether more realistic transverse coefficients can be obtained by defining more complex PUC's (Edwards et al. 1991) is still an open research question. As to the transport coefficients an important result is that absorption enhances longitudinal dispersion (see Fig. 6). The reason for this is that the solute near the grain is retarded more than away from the grain and this leads to spreading of the solute, which translates itself in a larger longitudinal dispersion coefficient. However, the enhancement effect appears to be too small to be of practical significance. Non-linear adsorption $K\left(c^{(0)}\right)$, see also Eqs. 3.11 and 3.12 , can lead to self-sharpening or convective spreading on the large scale. Finally, local (pore-scale) dispersion, considered in this article, cannot be disregarded in describing macroscopic dispersion, because macroscopic dispersion consists of a reversible and irreversible 
contribution (Jha et al. 2009; Berentsen et al. 2005). The irreversible contribution is caused by pore-scale mixing and subsequent diffusion. At a heterogeneity scale of the order of meters or larger, fluid elements that took different paths are incompletely mixed by diffusion (as diffusion is slow on such a large scale) and this can be considered as partly reversible dispersion. A challenge for future study is to investigate whether homogenization can contribute to find a more accurate partition between reversible and irreversible dispersion.

\section{Conclusions}

- This article compares the Peclet number dependence of longitudinal and transverse dispersion coefficients obtained by homogenization in a PUC that consists of a sphere (circle) in a cube (square) with experimental data of dispersion in porous media. We use the same porosity dependence as in the engineering literature. There is good agreement for longitudinal dispersion. The computed transverse dispersion coefficients for such a simple unit cell are much lower than experimental values.

- A slightly modified and simplified approach for homogenization shows that one of the assumptions is that the local spatial derivatives are one order of magnitude larger than the global derivatives. For the PUC of choice, the dispersion relations are identical to the relations obtained for periodic media. COMSOL can be readily used to compute longitudinal and transverse dispersion coefficients in $2 \mathrm{D}$ and $3 \mathrm{D}$. The $3 \mathrm{D}$ results are for the first time obtained at relevant Peclet numbers.

- Adsorption does not affect the transverse dispersion coefficient. However, adsorption enhances the longitudinal dispersion coefficient in agreement with an analysis of homogenization applied to Taylor dispersion discussed in the literature. The enhancement, even at retardation factors of 10 , is small.

Acknowledgments This article is the result of the Master Thesis work of Aiske Rijnks. In an early stage, we had a three-day discussion with Marjan Smit, Andrea Cortis, Ruud Schotting, and Jacob Bear leading to an inventory of the physical assumptions underlying homogenization. We thank Hamidreza Salimi for many useful suggestions. We would like to acknowledge Fred Vermolen for valuable suggestions on the numerical implementation. Furthermore, we thank Sorin Pop for contributing to the discussion on the physical perspective of homogenization, and for reading this manuscript. We thank Florian Kleinendorst (COMSOL) for his suggestion to use the appropriate Neumann condition in the periodic boundary conditions. An unexpected meeting with Pierre Tardif d'Hamonville during a Marie-Curie programme (GRASP) sponsored workshop on $\mathrm{CO}_{2}$ sequestration led to enlightening discussions. Finally, countless excellent comments of the referees and audiences in oral presentations have greatly contributed to this article. The study described here was supported by The Netherlands Organization for Scientific Research (NWO) for the project "Solubility/mobility of arsenic under changing redox conditions" (2001-2004).

Open Access This article is distributed under the terms of the Creative Commons Attribution Noncommercial License which permits any noncommercial use, distribution, and reproduction in any medium, provided the original author(s) and source are credited.

\section{Appendix A: The Flow Equations and Boundary Condition with Terms of Order $\varepsilon^{0}$ and $\varepsilon^{1}$}

This appendix derives the zeroth order transport equation from the terms of order $\varepsilon^{0}$ in Eq. 3.4 and the terms of order $\varepsilon^{1}$ in boundary condition (3.5) Eq. 3.4 is repeated here for convenience

$$
\frac{\partial c^{(0)}}{\partial t}+\operatorname{div}_{\mathrm{b}}\left(\mathbf{v} c^{(0)}\right)+\operatorname{div}_{\mathrm{s}}\left(\mathbf{v} c^{(1)}\right)=\frac{1}{P e} \operatorname{div}_{\mathrm{s}}\left(\operatorname{grad}_{\mathrm{b}} c^{(0)}\right)+\frac{1}{P e} \operatorname{div}_{\mathrm{s}}\left(\operatorname{grad}_{\mathrm{s}} c^{(1)}\right),
$$


where we used that $\operatorname{grad}_{\mathrm{s}} c^{(0)}=0$ because $c^{(0)}$ is constant on the microscale. It turns out that $\operatorname{div}_{\mathrm{s}}\left(\operatorname{grad}_{\mathrm{b}} c^{(0)}\right)$ cannot be taken equal to zero as it plays a role as part of the driving force for $c^{(1)}$, see Eq. B.1. In the same way, we collect the terms of order $\varepsilon^{1}$ in Eq. 3.5,

$$
\frac{1}{P e}\left(\operatorname{grad}_{\mathrm{b}} c^{(0)}\right) \cdot \mathbf{n}+\frac{1}{P e}\left(\operatorname{grad}_{\mathrm{s}} c^{(1)}\right) \cdot \mathbf{n}=-\frac{K\left(c^{(0)}\right)}{\varepsilon}\left(\frac{\partial c^{(0)}}{\partial t}\right)_{\Gamma} \text { at } \Gamma,
$$

where $K\left(c^{(0)}\right) / \varepsilon$ is of order $\varepsilon^{0}$.

An important step in homogenization is averaging: a quantity $Q$ is integrated over the fluid domain $\Omega_{1}$ and then divided by the total volume of the PUC, $|\Omega|$, represents the averaged behavior $\langle Q\rangle=\frac{1}{|\Omega|} \int_{\Omega_{1}} Q \mathrm{~d} \mathbf{r}_{\mathrm{s}}$, where the local scale coordinate $\mathbf{r}_{\mathrm{s}}=\left(x_{\mathrm{s}}, y_{\mathrm{s}}, z_{\mathrm{s}}\right)$ denotes a point in $\Omega_{1}$. After this averaging step, the quantity $\langle Q\rangle$ is only a function of the global scale coordinate $\mathbf{r}_{\mathrm{b}}=\left(x_{\mathrm{b}}, y_{\mathrm{b}}, z_{\mathrm{b}}\right)$. Applying this averaging procedure to Eq. 3.10 gives

$$
\begin{aligned}
& \frac{1}{|\Omega|} \int_{\Omega_{1}} \frac{\partial c^{(0)}}{\partial t} \mathrm{~d} \mathbf{r}_{\mathrm{s}}+\frac{1}{|\Omega|} \operatorname{div}_{\mathrm{b}}\left(\int_{\Omega_{1}} \mathbf{v} c^{(0)} \mathrm{d} \mathbf{r}_{\mathrm{s}}\right)+\frac{1}{|\Omega|} \int_{\Omega_{1}} \operatorname{div}_{\mathrm{s}}\left(\mathbf{v} c^{(1)}\right) \mathrm{d} \mathbf{r}_{\mathrm{s}} \\
= & \frac{1}{|\Omega|} \frac{1}{P e} \int_{\Omega_{1}} \operatorname{div}_{\mathrm{s}}\left(\operatorname{grad}_{\mathrm{b}} c^{(0)}\right) \mathrm{d} \mathbf{r}_{\mathrm{s}}+\frac{1}{|\Omega|} \frac{1}{P e} \int_{\Omega_{1}} \operatorname{div}_{\mathrm{s}}\left(\operatorname{grad}_{\mathrm{s}} c^{(1)}\right) \mathrm{dr}_{\mathrm{s}},
\end{aligned}
$$

where $\operatorname{div}_{\mathrm{b}}$ is not dependent on the small scale coordinate $\mathbf{r}_{\mathrm{s}}$ and is therefore taken outside the integral.

Application of the divergence theorem of Gauss converts the volume integral of the divergence of a vector $\mathbf{w}$ in a periodic domain into a surface integral in the following manner:

$$
\int_{\Omega_{1}} \operatorname{div}_{\mathrm{s}} \mathbf{w} \mathrm{d} \mathbf{r}_{\mathrm{s}}=\int_{\Gamma} \mathbf{w} \cdot \mathbf{n} \mathrm{d} s+\int_{\partial \Omega} \mathbf{w} \cdot \mathbf{n} \mathrm{d} s=\int_{\Gamma} \mathbf{w} \cdot \mathbf{n} \mathrm{d} s
$$

where we use that the surface integral over the outer boundary vanishes due to the periodicity of the unit cell. Applying Gauss's theorem to Eq. A.3 leads to

$$
\left\langle\frac{\partial c^{(0)}}{\partial t}\right\rangle+\operatorname{div}_{\mathrm{b}}\left\langle\mathbf{v} c^{(0)}\right\rangle=\frac{1}{|\Omega| P e} \int_{\Gamma}\left(\operatorname{grad}_{\mathrm{b}} c^{(0)}\right) \cdot \mathbf{n} \mathrm{d} s+\frac{1}{|\Omega| P e} \int_{\Gamma}\left(\operatorname{grad} c^{(1)}\right) \cdot \mathbf{n} \mathrm{d} s .
$$

Application of Gauss's theorem to the third term in Eq. A.3 shows that this term is zero, because of the $\Omega$ periodicity of $\mathbf{v} c^{(1)}$ and the no-slip condition $\mathbf{v}=0$ on the grain surface $\Gamma$. Note that $|\Omega|=1$, but we like to keep it for more transparent conversion to the full dimensional model in Sect. 4.2.

For the boundary condition Eq. 3.14 another procedure applies: integration of the boundary condition over the grain boundary leads to

$$
\int_{\Gamma} \frac{1}{P e} \operatorname{grad}_{\mathrm{b}} c^{(0)} \cdot \mathbf{n} \mathrm{d} s+\int_{\Gamma} \frac{1}{P e} \operatorname{grad}_{\mathrm{s}} c^{(1)} \cdot \mathbf{n} \mathrm{d} s=-\int_{\Gamma} \frac{K\left(c^{(0)}\right)}{\varepsilon}\left(\frac{\partial c^{(0)}}{\partial t}\right){ }_{\Gamma} \mathrm{d} s \quad \text { at } \Gamma .
$$


Substitution of this boundary condition in Eq. A.5 leads to

$$
\left\langle\frac{\partial c^{(0)}}{\partial t}\right\rangle+\operatorname{div}_{\mathrm{b}}\left\langle\mathbf{v} c^{(0)}\right\rangle=-\frac{1}{|\Omega|} \int_{\Gamma} \frac{K\left(c^{(0)}\right)}{\varepsilon}\left(\frac{\partial c^{(0)}}{\partial t}\right)_{\Gamma} \mathrm{d} s .
$$

We recall that $c^{(0)}$ is independent of the small scale coordinate $\mathbf{r}_{\mathrm{S}}$ and is therefore constant over the PUC. Therefore, we can take the terms $\frac{K\left(c^{(0)}\right)}{\varepsilon}\left(\frac{\partial c^{(0)}}{\partial t}\right)_{\Gamma}$ outside the integral. We obtain

$$
\left\langle\frac{\partial c^{(0)}}{\partial t}\right\rangle+\operatorname{div}_{\mathrm{b}}\left\langle\mathbf{v} c^{(0)}\right\rangle=-\frac{K\left(c^{(0)}\right)}{|\Omega| \varepsilon} \frac{\partial c^{(0)}}{\partial t} \int_{\Gamma} \mathrm{d} s=-\frac{K\left(c^{(0)}\right)}{\varphi|\Omega| \varepsilon}\left\langle\frac{\partial c^{(0)}}{\partial t}\right\rangle|\Gamma|,
$$

where $|\Gamma|$ denotes the surface area of the grain boundary. Collecting the terms with $\left\langle\frac{\partial c^{(0)}}{\partial t}\right\rangle$ we obtain

$$
\left\langle\frac{\partial c^{(0)}}{\partial t}\right\rangle\left(1+\frac{K\left(c^{(0)}\right)}{|\Omega| \varphi \varepsilon}|\Gamma|\right)+\operatorname{div}_{\mathrm{b}}\left\langle\mathbf{v} c^{(0)}\right\rangle=0 .
$$

This is the reactive transport equation, which is often used to describe adsorption-convection transport problems in practice (Bear 1972). The factor $\frac{K\left(c^{(0)}\right)}{|\Omega| \varphi \varepsilon}|\Gamma|=\frac{\frac{\delta}{\ell}\left(\frac{\partial c_{\mathrm{a}}}{\partial c}\right)_{c^{(0)}}}{\mid \Omega(\varphi)}|\Gamma|$ describes the ratio of the adsorbed mass divided by the mass of the free concentration.

For the following it is useful to rewrite Eq. A.9 in a non-averaged form, i.e.,

$$
\varphi \frac{\partial c^{(0)}}{\partial t}\left(1+\frac{K\left(c^{(0)}\right)}{|\Omega| \varphi \varepsilon}|\Gamma|\right)+\varphi \overline{\mathbf{v}} \cdot \operatorname{grad}_{\mathrm{b}} c^{(0)}=0,
$$

where we use $\left\langle\frac{\partial c^{(0)}}{\partial t}\right\rangle=\frac{1}{|\Omega|} \frac{\partial c^{(0)}}{\partial t} \int_{\Omega_{1}} d \mathbf{r}_{\mathrm{s}}=\frac{\Omega_{1}}{|\Omega|} \frac{\partial c^{(0)}}{\partial t}=\varphi \frac{\partial c^{(0)}}{\partial t}$. Therefore, $\frac{\partial c^{(0)}}{\partial t}=\frac{1}{\varphi}\left\langle\frac{\partial c^{(0)}}{\partial t}\right\rangle$. We applied the product rule of differentiation for the second term in Eq. A.8, the incompressibility condition $\operatorname{div}_{\mathrm{s}} \mathbf{v}=\operatorname{div}_{\mathrm{b}} \mathbf{v}=0$ and we define $\overline{\mathbf{v}}=\frac{1}{\left|\Omega_{1}\right|} \int_{\Omega_{1}} \mathbf{v} d r_{\mathrm{s}}$, where $\left|\Omega_{1}\right|$ denotes the volume of the fluid domain. Tortuosity effects do not enter in this integration and therefore $\bar{v}$ represents the interstitial velocity, i.e., the Darcy velocity divided by the porosity. For reasons of concise notation, we use the retardation factor $R=1+\frac{K\left(c^{(0)}\right)}{|\Omega| \varphi \varepsilon}|\Gamma|>1$ or $\frac{K\left(c^{(0)}\right)}{|\Omega| \varphi \varepsilon}|\Gamma|=R-1$ and obtain

$$
\frac{\partial c^{(0)}}{\partial t}+\frac{\overline{\mathbf{v}}}{R} \cdot \operatorname{grad}_{\mathrm{b}} c^{(0)}=0 .
$$

\section{Appendix B: Derivation of the Cell Equation}

This Appendix derives the cell equation (3.13) and the corresponding grain boundary condition (3.15), which makes it possible to find the first order correction to the concentration. To eliminate the time derivative from Eq. 3.10, we subtract Eq. 3.11 from Eq. 3.10 and obtain

$$
\left(\mathbf{v}-\frac{\overline{\mathbf{v}}}{R}\right) \cdot \operatorname{grad}_{\mathrm{b}} c^{(0)}+\operatorname{div}_{\mathrm{s}}\left(\mathbf{v} c^{(1)}\right)=\frac{1}{P e} \operatorname{div}_{\mathrm{s}}\left(\operatorname{grad}_{\mathrm{b}} c^{(0)}\right)+\frac{1}{P e} \operatorname{div}_{\mathrm{s}}\left(\operatorname{grad}_{\mathrm{s}} c^{(1)}\right) .
$$


The global gradient $\operatorname{grad}_{\mathrm{b}} c^{(0)}$ is acting as a driving force for the local flow and the local concentration field $c^{(1)}$ is proportional to $\operatorname{grad}_{\mathrm{b}} c^{(0)}$. If $\operatorname{grad}_{\mathrm{b}} c^{(0)}$ is applied also in the $x$-direction we obtain the longitudinal dispersion coefficient. If we apply it in the $y$-direction or $z$-direction we obtain the transverse dispersion coefficient. For our unit cell there should be no difference to apply either the pressure gradient or the concentration gradient in an arbitrary direction to obtain one of the components of the dispersion tensor, but it can be expected that an arbitrary direction gives problems in the numerical calculations.

In order to proceed, we use that Eq. B.1 is linear and therefore that $c^{(1)}$ is linearly related to $\operatorname{grad}_{\mathrm{b}} c^{(0)}$ (Sanchez-Palencia 1980), which in its most general form can be written as $c^{(1)}=\vec{\chi} \cdot \operatorname{grad}_{\mathrm{b}} c^{(0)}$, where $\vec{\chi}$ is a vector. Note that the vector $\vec{\chi}(x, y, z)$ also depends on the space coordinates. Substitution of $c^{(1)}=\vec{\chi} \cdot \operatorname{grad}_{\mathrm{b}} c^{(0)}$ in Eq. B.1 leads to

$$
\begin{aligned}
& \left(\mathbf{v}-\frac{\overline{\mathbf{v}}}{R}\right) \cdot \operatorname{grad}_{\mathrm{b}} c^{(0)}+\operatorname{div}_{\mathrm{s}}\left(\mathbf{v} \otimes \vec{\chi} \cdot \operatorname{grad}_{\mathrm{b}} c^{(0)}\right) \\
& =\frac{1}{P e} \operatorname{div}_{\mathrm{s}}\left(\left(\mathbf{I}+\operatorname{grad}_{\mathrm{s}} \otimes \vec{\chi}\right) \cdot \operatorname{grad}_{\mathrm{b}} c^{(0)}\right),
\end{aligned}
$$

where we use the notation $(\mathbf{v} \otimes \vec{\chi})$ for the product between the two vectors $\mathbf{v}$ and $\vec{\chi}$ and $\operatorname{grad}_{\mathrm{s}} \otimes \vec{\chi}$ for the product between the two vectors $\operatorname{grad}_{\mathrm{s}}$ and $\vec{\chi}$. This product is called the dyadic product. The dyadic product is a tensor with as elements, e.g., on the $(1,2)$ position the $x$-component of one vector with the $y$-component of the other vector. Note that $\mathbf{v} \otimes \vec{\chi} \neq \vec{\chi} \otimes \mathbf{v}$. We use I to denote the unit tensor. This equation can be used to evaluate the dispersion tensor. Indeed in Eq. B.2, $\left(\mathbf{v}-\frac{\overline{\mathbf{v}}}{R}\right) \cdot \operatorname{grad}_{\mathrm{b}} c^{(0)}$ is the convective/reaction/adsorption transport term, $\operatorname{div}_{\mathrm{s}}\left((\mathbf{v} \otimes \vec{\chi}) \cdot \operatorname{grad}_{\mathrm{b}} c^{(0)}\right)$ the dispersion term and $\frac{1}{P e} \operatorname{div}_{\mathrm{s}}\left(\left(\mathbf{I}+\operatorname{grad}_{\mathrm{s}} \otimes \vec{\chi}\right) \cdot \operatorname{grad}_{\mathrm{b}} c^{(0)}\right)$ the diffusive transport term.

Equation B.2 is an equation for the PUC scale. Therefore, we need to eliminate $\operatorname{grad}_{\mathrm{b}} c^{(0)}$ from Eq. B.2. The term $\operatorname{grad}_{\mathrm{b}} c^{(0)}$ can be considered a constant vector on the small scale, because of the disparity of scales. By making various choices we can obtain the components of $\vec{\chi}$. For instance, we consider the longitudinal (flow) direction $\operatorname{grad}_{\mathrm{b}} c^{(0)}=-\mathbf{e}_{x}$, where we use the minus sign because the concentration decreases in the longitudinal direction. The $x$-component of the vector $\vec{\chi}$ is denoted by $\chi_{x}=\vec{\chi} \cdot \mathbf{e}_{x}$, and therefore $\vec{\chi} \cdot \operatorname{grad}_{\mathrm{b}} c^{(0)}=$ $-\chi_{x}$. We can use $\chi_{x}$ to obtain the first order correction $c^{(1)}$ to the concentration $c^{(0)}$ when the system is subjected to a unit global gradient in the $x$-direction. The behavior of $\chi_{x}$ is therefore a measure for the concentration fluctuations caused by dispersion as a result of the non-homogeneous nature of a porous medium. Next, we use that $(\mathbf{v} \otimes \vec{\chi}) \cdot \operatorname{grad}_{\mathrm{b}} c^{(0)}=-\chi_{x} \mathbf{v}$ and $\left(\operatorname{grad}_{\mathrm{s}} \otimes \vec{\chi}\right) \cdot \operatorname{grad}_{\mathrm{b}} c^{(0)}=-\operatorname{grad}_{\mathrm{s}} \chi_{x} \cdot$ Moreover, $\mathbf{I} \cdot \operatorname{grad}_{\mathrm{b}} c^{(0)}=-\mathbf{e}_{x}=-\operatorname{grad}_{\mathrm{s}} x_{\mathrm{s}}$. Substitution of $\operatorname{grad}_{\mathrm{b}} c^{(0)}=-\mathbf{e}_{x}$ in Eq. B.2 leads therefore to the source, convection-diffusion equation

$$
-\frac{\bar{v}_{x}}{R}+\operatorname{div}_{\mathrm{s}}\left(\mathbf{v}\left(\chi_{x}+x_{\mathrm{s}}\right)\right)=\frac{1}{P e} \operatorname{div}_{\mathrm{s}} \operatorname{grad}_{\mathrm{s}}\left(\chi_{x}+x_{\mathrm{s}}\right) .
$$

The velocity $\mathbf{v}$ is the mass averaged velocity on the "Stokes" scale (see Sect. 2).

Substitution of $c^{(1)}=\vec{\chi} \cdot \operatorname{grad}_{\mathrm{b}} c^{(0)}$ in boundary condition Eq. 3.14, combining with Eq. 3.11 and looking at the case $\operatorname{grad}_{\mathrm{b}} c^{(0)}=-\mathbf{e}_{x}$ leads with the relation between $K\left(c^{(0)}\right)$ and the retardation factor $R$ defined above Eq. 3.11, i.e., $\frac{K\left(c^{(0)}\right)}{|\Omega| \varphi \varepsilon}|\Gamma|=R-1$ to

$$
\frac{1}{P e}\left(\operatorname{grad}_{\mathrm{s}}\left(\chi_{x}+x_{\mathrm{s}}\right)\right) \cdot \mathbf{n}=\frac{K\left(c^{(0)}\right)}{\varepsilon} \frac{\bar{v}_{x}}{R}=\frac{(R-1)|\Omega|}{R|\Gamma|} \varphi \bar{v}_{x} \quad \text { at } \Gamma,
$$


where we used again that $\left(\operatorname{grad}_{\mathrm{s}} \otimes \vec{\chi}\right) \cdot \operatorname{grad}_{\mathrm{b}} c^{(0)}=-\operatorname{grad}_{\mathrm{s}} \chi_{x}$ and $\operatorname{grad}_{\mathrm{b}} c^{(0)}=-\mathbf{e}_{x}=$ $-\operatorname{grad}_{\mathrm{s}} x_{\mathrm{s}}$. Note that $|\Gamma|$ is the dimensionless surface area of the grain, i.e., in the PUC with a volume equal to one.

We can calculate the local concentration variations of the first order term with the help of a numerical method. In this study, we use the finite element software package COMSOL to evaluate Eqs. (3.13) and BC (3.15) to solve for $\chi_{x}$.

\section{Appendix C: The Equations with Terms of Order $\varepsilon^{1}$ and $\varepsilon^{2}$}

This Appendix derives the diffusion-convection equation (3.16) for the first order concentration term, which can be used to find the upscaled diffusion-convection equation and to derive the dispersion terms. A higher order dimensionless convection-diffusion equation and boundary condition lead to a more accurate description than Eq. 3.11. We derive these equations in the same way as the lower order equations. The equation with terms of order $\varepsilon^{1}$ from Eq. 3.4 is

$$
\begin{aligned}
& \frac{\partial c^{(1)}}{\partial t}+\operatorname{div}_{\mathrm{b}}\left(\mathbf{v} c^{(1)}\right)+\operatorname{div}_{\mathrm{s}}\left(\mathbf{v} c^{(2)}\right)=\frac{1}{P e} \operatorname{div}_{\mathrm{b}}\left(\operatorname{grad}_{\mathrm{b}} c^{(0)}\right)+\frac{1}{P e} \operatorname{div}_{\mathrm{s}}\left(\operatorname{grad}_{\mathrm{b}} c^{(1)}\right) \\
& +\frac{1}{P e} \operatorname{div}_{\mathrm{b}}\left(\operatorname{grad}_{\mathrm{s}} c^{(1)}\right)+\frac{1}{P e} \operatorname{div}_{\mathrm{s}}\left(\operatorname{grad}_{\mathrm{s}} c^{(2)}\right) .
\end{aligned}
$$

Application of the averaging procedure and Eq. A.4 leads to

$$
\begin{aligned}
& \left\langle\frac{\partial c^{(1)}}{\partial t}\right\rangle+\operatorname{div}_{\mathrm{b}}\left\langle\mathbf{v} c^{(1)}\right\rangle=\frac{1}{P e} \operatorname{div}_{\mathrm{b}}\left\langle\operatorname{grad}_{\mathrm{b}} c^{(0)}\right\rangle+\frac{1}{|\Omega| P e} \int_{\Gamma} \operatorname{grad}_{\mathrm{b}} c^{(1)} \cdot \mathbf{n} \mathrm{d} s \\
& +\frac{1}{P e} \operatorname{div}_{\mathrm{b}}\left\langle\operatorname{grad}_{\mathrm{s}} c^{(1)}\right\rangle+\frac{1}{|\Omega| P e} \int_{\Gamma} \operatorname{grad}_{\mathrm{s}} c^{(2)} \cdot \mathbf{n} \mathrm{d} s,
\end{aligned}
$$

where $\int_{\Gamma}\left(\mathbf{v} c^{(2)}\right) \cdot \mathbf{n} \mathrm{d} s=0$ because of no-slip conditions and both $\mathbf{d i v}_{\mathrm{b}}$ and $\operatorname{grad}_{\mathrm{b}}$ are independent of the local scale and therefore not subjected to the averaging procedure. We note that $|\Omega|=1$, but it will play a role when we are transforming to full dimensional equations in Sect. 4.2.

The boundary condition with terms of order $\varepsilon^{2}$ from Eq. 3.5 is

$$
\frac{1}{P e}\left(\operatorname{grad}_{\mathrm{b}} c^{(1)}\right) \cdot \mathbf{n}+\frac{1}{P e}\left(\operatorname{grad}_{\mathrm{s}} c^{(2)}\right) \cdot \mathbf{n}=-\frac{K\left(c^{(0)}\right)}{\varepsilon}\left(\frac{\partial c^{(1)}}{\partial t}\right)_{\Gamma} \text { at } \Gamma .
$$

After integration over the grain boundary this equation becomes

$$
\int_{\Gamma} \frac{1}{P e} \operatorname{grad}_{\mathrm{b}} c^{(1)} \cdot \mathbf{n} \mathrm{d} s+\int_{\Gamma} \frac{1}{P e} \operatorname{grad}_{\mathrm{s}} c^{(2)} \cdot \mathbf{n} \mathrm{d} s=-\int_{\Gamma} \frac{K\left(c^{(0)}\right)}{\varepsilon}\left(\frac{\partial c^{(1)}}{\partial t}\right)_{\Gamma} \mathrm{d} s \quad \text { at } \Gamma .
$$

Substitution of the boundary condition (C.4) into Eq. C.2 leads to the complete higher order convection-diffusion equation, i.e.,

$\left\langle\frac{\partial c^{(1)}}{\partial t}\right\rangle+\operatorname{div}_{\mathrm{b}}\left\langle\mathbf{v} c^{(1)}\right\rangle=\frac{1}{P e} \operatorname{div}_{\mathrm{b}}\left(\left\langle\operatorname{grad}_{\mathrm{b}} c^{(0)}\right\rangle+\left\langle\operatorname{grad}_{\mathrm{s}} c^{(1)}\right\rangle\right)-\int_{\Gamma} \frac{K\left(c^{(0)}\right)}{|\Omega| \varepsilon}\left(\frac{\partial c^{(1)}}{\partial t}\right)_{\Gamma} \mathrm{d} s$. 


\section{References}

Allaire, G., Brizzi, R., Mikelic, A., Piatnitski, A.: Two-scale expansion with drift approach to the Taylor dispersion for reactive transport through porous media. Chem. Eng. Sci. 65(7), 2292-2300 (2010a)

Allaire, G., Mikelic, A., Piatnitski, A.: Homogenization approach to the dispersion theory for reactive transport through porous media. SIAM J. Math. Anal. 42(1), 125-144 (2010b)

Arya, A., Hewett, T.A., Larson, R.G., Lake, L.W.: Dispersion and reservoir heterogeneity. SPE Reserv. Eng. 3(1), 139-148 (1988)

Attinger, S., Dimitrova, J., Kinzelbach, W.: Homogenization of the transport behavior of nonlinearly adsorbing pollutants in physically and chemically heterogeneous aquifers. Adv. Water Resour. 32(5), 767-777 (2009)

Auriault, J.L.: Heterogeneous medium: is an equivalent macroscopic description possible. Int. J. Eng. Sci. 29(7), 785-795 (1991)

Auriault, J.L.: Upscaling heterogeneous media by asymptotic expansions. J. Eng. Mech. ASCE 128(8), 817-822 (2002)

Auriault, J.L., Adler, P.M.: Taylor dispersion in porous-media-analysis by multiple scale expansions. Adv. Water Resour. 18(4), 217-226 (1995)

Auriault, J.L., Lewandowska, J.: Homogenization analysis of diffusion and adsorption macrotransport in porous-media-macrotransport in the absence of advection. Geotechnique 43(3), 457-469 (1993)

Auriault, J.L., Lewandowska, J.: Diffusion/adsorption/advection macrotransport in soils. Eur. J. Mech. A 15(4), 681-704 (1996)

Auriault, J.L., Lewandowska, J.: Modelling of pollutant migration in porous media with interfacial transfer: local equilibrium/non-equilibrium. Mech. Cohesive-Frict. Mater. 2(3), 205-221 (1997)

Auriault, J.L., Lewandowska, J.: Upscaling: cell symmetries and scale separation. Transp. Porous Media 43(3), 473-485 (2001)

Auriault, J.L., Royer, P.: Double conductivity media - a comparison between phenomenological and homogenization approaches. Int. J. Heat Mass Transf. 36(10), 2613-2621 (1993a)

Auriault, J.L., Royer, P.: Gas-flow through a double-porosity porous-medium. Comptes Rendus De L' Academie Des Sciences Serie Ii 317(4), 431-436 (1993b)

Auriault, J.L., Bouvard, D., Dellis, C., Lafer, M.: Modeling of hot compaction of metal-powder by homogenization. Mech. Mater. 13(3), 247-255 (1992)

Auriault, J.L., Geindreau, C., Boutin, C.: Filtration law in porous media with poor separation of scales. Transp. Porous Media 60(1), 89-108 (2005)

Auriault, J.L., Boutin, C., Geindreau, C.: Homogenization of Coupled Phenomena in Heterogenous Media. Wiley, Hoboken (2009)

Bachmat, Y., Bear, J.: The dispersive flux in transport phenomena in porous-media. Adv. Water Resour. 6(3), 169-174 (1983)

Bear, J.: Dynamics of Fluids in Porous Media. Dover Publications Inc., New York (1972)

Bensoussan, A., Lions, J.L., Papanicolaou, G.: Asymptotic Analysis for Periodic Structures. NorthHolland, Amsterdam (1978)

Berentsen, C.W.J., Verlaan, M.L., Kruijsdijk, C.Van : Upscaling and reversibility of Taylor dispersion in heterogeneous porous media. Phys. Rev. E 71(4), 46308 (2005)

Bhatt, A.M., Lourma, S., Donselaar, R., Bruining, J.: Geologic origin of arsenic contamination along the Sone and Ganges Rivers, Bihar, India. In: Royal Geographical Society-IBG Annual International Conference 2011, Water Scarcity in Developing Economies: Issues and Solutions, volume abstract, London (2011)

Bhattacharya, R.N., Gupta, V.K.: A theoretical explanation of solute dispersion in saturated porous media at the Darcy scale. Water Resour. Res. 19, 938-944 (1983)

Bhattacharya, R.N., Gupta, V.K., Walker, H.F.: Asymptotics of solute dispersion in periodic porous media. SIAM J. Appl. Math. 49(1), 86-98 (1989)

Bird, R.B., Stewart, W.E., Lightfoot, E.N.: Transport Phenomena. Wiley, New York (1960)

Bouddour, A., Auriault, J.L., Mhamdi-Alaoui, M.: Erosion and deposition of solid particles in porous media: homogenization analysis of a formation damage. Transp. Porous Media 25(2), 121-146 (1996)

Bourgeat, A., Piatnitski, A.: Approximations of effective coefficients in stochastic homogenization. Annales de l'Institut Henri Poincaré/Probabilités Et Statistiques 40(2), 153-165 (2004)

Bourgeat, A., Quintard, M., Whitaker, S.: Comparison between homogenization theory and volume averaging method with closure problem. Comptes Rendus De L' Academie Des Sciences Serie Ii 306(7), 463-466 (1988)

Brenner, H.: Dispersion resulting from flow through spatially periodic porous media. Philos. Trans. R. Soc. Lond. A 297(1430), 81-133 (1980) 
Bruining, J., Darwish, M.I.M.: Homogenizeation for $\mathrm{Fe}^{2+}$ deposition near drink water tube wells during arsenic remediation. In: European Conference on the Mathematics of Oil Recovery X, Amsterdam, p. 3 (2006)

Buyuktas, D., Wallender, W.W.: Dispersion in spatially periodic porous media. Heat Mass Transf. 40(3), 261-270 (2004)

Carbonnell, R.G., Whitaker, S.: Dispersion in pulsed systems. II. Theoretical developments for passive dispersion in porous media. Chem. Eng. Sci. 38(11), 1795-1802 (1983)

Chrysikopoulos, C.V., Kitanidis, P.K., Roberts, P.V. Generalized Taylor-Aris moment analysis of the transport of sorbing solutes through porous media with spatially-periodic retardation factor. Transp. Porous Media 7, 163-185 (1992)

Dagan, G., Neuman, S.P.: Subsurface Flow and Transport: A Stochastic Approach. Cambridge University Press, Cambridge (1997)

Darwish, M.I.M., Van Beek, V.M., Bruining, J.: Visualization experiments of iron precipitates: application for in-situ arsenic remediation. J. Geochem. Explor. 88(1-3), 19-23 (2006)

Delgado, J.M.P.Q.: A critical review of dispersion in packed beds. Heat Mass Transf. 42(4), 279-310 (2006)

Delgado, J.M.P.Q.: Longitudinal and transverse dispersion in porous media. Trans. IChemE A 85(A9), 12451252 (2007)

Didierjean, S., Souto, H.P.A., Delannay, R., Moyne, C.: Dispersion in periodic porous media. Experience versus theory for two-dimensional systems. Chem. Eng. Sci. 52(12), 1861-1874 (1997)

Dullien, F.A.L.: Porous Media-Fluid Transport and Pore Structure. Academic Press, New York (1992)

Durlofsky, L.J.: Numerical calculation of equivalent grid block permeability tensors for heterogeneous porous media. Water Resour. Res. 27(5), 699-708 (1991)

Edwards, D.A., Shapiro, M., Brenner, H., Shapira, M.: Dispersion of inert solutes in spatially periodic, 2-dimensional model porous-media. Transp. Porous Media 6(4), 337-358 (1991)

Eidsath, A., Whitaker, S., Carbonell, R.G., Herrmann, L.R.: Dispersion in pulsed systems. Pt. 3: Comparison between theory and experiments for packed beds. Chem. Eng. Sci. 38(11), 1803-1816 (1983)

Fannjiang, A., Papanicolaou, G.: Convection-enhanced diffusion for random flows. J. Stat. Phys. 88(5), 10331076 (1996)

Gelhar, L.W.: Stochastic Subsurface Hydrology. Prentice Hall, Englewood Cliffs (1993)

Gupta, V.K., Bhattacharya, R.N.: Solute dispersion in multidimensional periodic saturated porus media. Water Resour. Res. WRERAO 22(2), 156-164 (1986)

Hornung, U.: Homogenization and Porous Media. Springer, New York (1997)

Jha, R.K., John, A.K., Bryant, S.L., Lake, L.W.: Flow reversal and mixing. SPE J. 14, 41-49 (2009)

Lowe, C.P., Frenkel, D.: Do hydrodynamic dispersion coefficients exist. Phys. Rev. Lett. 77(22), 4552-4555 (1996)

Marle, C.M.: On macroscopic equations governing multiphase flow with diffusion and chemical reactions in porous media. Int. J. Eng. Sci. 20(5), 643-662 (1982)

Mauri, R.: Dispersion, convection, and reaction in porous media. Phys. Fluids A 3, 743-756 (1991)

Mikelic, A., Rosier, C.: Modeling solute transport through unsaturated porous media using homogenization I. Comput. Appl. Math. 23, 195-211 (2004)

Moralles-Wilhelm, F., Gelhar, L.: Stochastic analysis of sorption macrokinetics in heterogeneous aquifers. Water Resour. Res. 32(6), 1541-1549 (1996)

Perkins, T.K., Dallas, T.E.X.: A review of diffusion and dispersion in porous media. SPE J. 3, 70-80 (1962)

Rajaram, H., Gelhar, L.W.: Plume-scale dependent dispersion in heterogeneous aquifers 1. Lagrangian analysis in a stratified aquifer. Water Resour. Res. 29(9), 3249-3260 (1993)

Rajaram, H., Gelhar, L.W.: Plume-scale dependent dispersion in aquifers with a wide range of scales of heterogeneity. Water Resour. Res. 31(10), 2469-2482 (1995)

Rhee, H.K., Aris, R., Amundson, N.R.: First-Order Partial Differential Equations, vol. 2. Dover Publications, New York (2001)

Roth, K., Jury, W.A.: Linear transport models for adsorbing solutes. Water Resour. Res. 29(4), 1195-1203 (1993)

Rubin, Y., Hubbard, S.S.: Hydrogeophysics, vol. 50. Springer, Dordrecht (2005)

Salimi, H., Bruining, H. Upscaling in vertically fractured oil reservoirs using homogenization. Transp. Porous Media 84, 21-53 (2010a)

Salimi, H., Bruining, J.: Improved prediction of oil recovery from waterflooded fractured reservoirs using homogenization. SPE Reserv. Eval. Eng. 13(1), 44-55 (2010b)

Sanchez-Palencia, E.: Non-homogeneous media and vibration theory. In: Non-Homogeneous Media and Vibration Theory, vol. 127. Springer, Berlin (1980)

Shook, M., Li, D., Lake, L.W.: Scaling immiscible flow through permeable media by inspectional analysis. In Situ 16(4), 311-349 (1992) 
Souto, H.P.A., Moyne, C.: Dispersion in two-dimensional periodic porous media. Part II. Dispersion tensor. Phys. Fluids 9, 2253-2263 (1997)

Tardif d'Hamonville, P., Ern, A., Dormieux, L.: Finite element evaluation of diffusion and dispersion tensors in periodic porous media with advection. Comput. Geosci. 11(1), 43-58 (2007)

Uffink, G., Elfeki, A., Dekking, M., Bruining, J., Kraaikamp, C.: Understanding the non-Gaussian nature of reactive solute transport. From particle dynamics to the partial differential equations. Arxiv preprint arXiv:1101.2510 (2011)

Van Duijn, C.J., Mikelic, A., Pop, I.S., Rosier, C.: Effective dispersion equations for reactive flows with dominant Peclet and Damköhler numbers. Adv. Chem. Eng. 34, 1-45 (2008)

Van Genuchten, M.Th., Wierenga, P.J.: Mass transfer studies in sorbing porous media. 1 Analytical solutions. J. Soil Sci. Soc. Am. 40(4), 473-480 (1976)

Whitaker, S.: The Method of Volume Averaging. Kluwer, Dordrecht (1999)

Wood, B.D.: The role of scaling laws in upscaling. Adv. Water Resour. 32(5), 723-736 (2009)

Wronski, S., Molga, E.: Axial dispersion in packed beds: the effect of particle size non-uniformities. Chem. Eng. Process. 22(3), 123-135 (1987)

Wygal, R.J.: Construction of models that simulate oil reservoirs. Soc. Pet. Eng. J. 3, 281-286 (1963)

Zhang, D.: Stochastic Methods for Flow in Porous Media: Coping with Uncertainties. Academic Press, San Diego (2002) 